\title{
Local dynamics and global attractivity of a certain second-order quadratic fractional difference equation
}

\author{
Mustafa RS Kulenović ${ }^{*}$, Esmir Pilav² and Enesa Silić ${ }^{3}$
}

\section{"Correspondence:}

kulenm@math.uri.edu

'Department of Mathematics,

University of Rhode Island, Kingston,

Rhode Island 02881-0816, USA

Full list of author information is

available at the end of the article

\begin{abstract}
We investigate the local and global character of the equilibrium and the local stability of the period-two solution of the difference equation $x_{n+1}=\frac{\beta x_{n} x_{n-1}+\gamma x_{n-1}^{2}+\delta x_{n}}{B x_{n} x_{n-1}+C X_{n-1}^{2}+D x_{n}}$ where the parameters $\beta, \gamma, \delta, B, C, D$ are nonnegative numbers which satisfy $B+C+D>0$ and the initial conditions $x_{-1}$ and $x_{0}$ are arbitrary nonnegative numbers such that $B x_{n} x_{n-1}+C x_{n-1}^{2}+D x_{n}>0$ for all $n \geq 0$.

MSC: Primary 39A10; 39A11; secondary 39A30
\end{abstract}

Keywords: boundedness; difference equation; global attractivity; local stability; period-two solutions

\section{Introduction and preliminaries}

In this paper we study the global dynamics of the following rational difference equation:

$$
x_{n+1}=\frac{\beta x_{n} x_{n-1}+\gamma x_{n-1}^{2}+\delta x_{n}}{B x_{n} x_{n-1}+C x_{n-1}^{2}+D x_{n}}, \quad n=0,1,2, \ldots
$$

where the parameters $\beta, \gamma, \delta, B, C, D$ are nonnegative numbers which satisfy $B+C+$ $D>0$ and the initial conditions $x_{-1}$ and $x_{0}$ are arbitrary nonnegative numbers such that $B x_{n} x_{n-1}+C x_{n-1}^{2}+D x_{n}>0$ for all $n \geq 0$.

Equation (1), which has been studied in [1-3], is a special case of a general second-order quadratic fractional equation of the form

$$
x_{n+1}=\frac{A x_{n}^{2}+B x_{n} x_{n-1}+C x_{n-1}^{2}+D x_{n}+E x_{n-1}+F}{a x_{n}^{2}+b x_{n} x_{n-1}+c x_{n-1}^{2}+d x_{n}+e x_{n-1}+f}, \quad n=0,1, \ldots
$$

with nonnegative parameters and initial conditions such that $A+B+C>0, a+b+c+$ $d+e+f>0$ and $a x_{n}^{2}+b x_{n} x_{n-1}+c x_{n-1}^{2}+d x_{n}+e x_{n-1}+f>0, n=0,1, \ldots$. Several global asymptotic results for some special cases of (2) were obtained in [4-7].

The change of variable $x_{n}=1 / u_{n}$ transforms (1) into the difference equation

$$
u_{n+1}=\frac{D u_{n-1}^{2}+C u_{n}+B u_{n-1}}{\delta u_{n-1}^{2}+\gamma u_{n}+\beta u_{n-1}}, \quad n=0,1, \ldots,
$$

O2014 Kulenović et al.; licensee Springer. This is an Open Access article distributed under the terms of the Creative Commons Attribution License (http://creativecommons.org/licenses/by/2.0), which permits unrestricted use, distribution, and reproduction in any medium, provided the original work is properly cited. 
where we assume that $\delta+\beta+\gamma>0$ and that the nonnegative initial conditions $u_{-1}, u_{0}$ are such that $\delta u_{n-1}^{2}+\gamma u_{n}+\beta u_{n-1}>0$ for all $n \geq 0$. Thus the results of this paper extend to (3).

The first systematic study of global dynamics of a special quadratic fractional case of (2) where $A=C=D=a=c=d=0$ was performed in $[1,2]$. The dynamics of some related quadratic fractional difference equations was considered in the papers [4-7]. In this paper we will perform the local stability analysis of the unique equilibrium and the period-two solution and we will give the necessary and sufficient conditions for the equilibrium to be locally asymptotically stable, a saddle point, a repeller or a non-hyperbolic equilibrium. The local stability analysis indicates that some possible dynamics scenarios for (1) include period-doubling bifurcations and Naimark-Sacker bifurcation and global attractivity of the equilibrium, see $[8,9]$. This means that the techniques we used in $[3,4,9-14]$ are applicable. We will also obtain the global asymptotic stability results for (1). As we have seen in [11] an efficient way of studying the dynamics of (1) is considering the dynamics of 49 special cases of (1) which are obtained when one or more coefficients are set to zero. Based on our results in [11], it is difficult to prove global asymptotic stability results of the unique equilibrium even for linear fractional difference equations; there are still two remaining cases one needs to study to prove the general conjecture that the local stability of the unique equilibrium implies the global stability.

Some interesting special cases of (1), which were thoroughly studied in [11], are the following equations.

(1) The Beverton-Holt difference equation when $\gamma=\delta=C=0$ :

$$
x_{n+1}=\frac{\beta x_{n-1}}{B x_{n-1}+D}, \quad n=0,1, \ldots
$$

which represents the basic discrete model in population dynamics, see [15].

(2) The Riccati difference equation when $\gamma=C=0$ :

$$
x_{n+1}=\frac{\beta x_{n-1}+\delta}{B x_{n-1}+D}, \quad n=0,1, \ldots
$$

(3) The difference equation studied in $[11,16,17]$, when $\delta=D=0$ :

$$
x_{n+1}=\frac{\beta x_{n}+\gamma x_{n-1}}{B x_{n}+C x_{n-1}}, \quad n=0,1, \ldots
$$

which represents the discretization of the differential equation model in biochemical networks, see [18].

The global attractivity results obtained specifically for the complicated cases of (2) are the following theorems [19].

Theorem 1 Assume that (2) has the unique equilibrium $\bar{x}$. If the following condition holds:

$$
\frac{(|A-a \bar{x}|+|B-b \bar{x}|+|C-c \bar{x}|)(U+\bar{x})+|D-d \bar{x}|+|E-e \bar{x}|}{(a+b+c) L^{2}+(d+e) L+f}<1,
$$

where $L$ and $U$ are lower and upper bounds of all solutions of (2) and $L+f>0$, then $\bar{x}$ is globally asymptotically stable. 
Theorem 2 Assume that (2) has the unique equilibrium $\bar{x}$ in the interval $[m, M]$, where $m=\min \left\{\bar{x}, x_{-1}, x_{0}\right\}$ and $M=\max \left\{\bar{x}, x_{-1}, x_{0}\right\}$ are lower and upper bounds of a specific solution of (2) and $m+f>0$. If the following condition holds:

$$
(|A-a \bar{x}|+|B-b \bar{x}|+|C-c \bar{x}|)(M+\bar{x})+|D-d \bar{x}|+|E-e \bar{x}|<(a+b+c) m^{2}+(d+e) m+f,
$$

then $\bar{x}$ is globally asymptotically stable on the interval $[m, M]$.

In the case of (1) Theorems 1 and 2 give the following special results.

Corollary 1 If the following condition holds:

$$
\frac{(|\beta-B \bar{x}|+|\gamma-C \bar{x}|)(U+\bar{x})+|\delta-D \bar{x}|}{(B+C) L^{2}+D L}<1
$$

where $L>0$ and $U$ are lower and upper bounds of all solutions of (1), then $\bar{x}$ is globally asymptotically stable.

Corollary 2 If the following condition holds:

$$
(|\beta-B \bar{x}|+|\gamma-C \bar{x}|)(M+\bar{x})+|\delta-D \bar{x}|<(B+C) m^{2}+D m,
$$

where $m=\min \left\{\bar{x}, x_{-1}, x_{0}\right\}>0$ and $M=\max \left\{\bar{x}, x_{-1}, x_{0}\right\}$ are lower and upper bounds of $a$ specific solution of (1), then the unique equilibrium $\bar{x}$ is globally asymptotically stable on the interval $[m, M]$.

In this paper we present the local stability analysis for the unique equilibrium and the period-two solutions of (1) and then we apply Corollaries 1 and 2 to some special cases of (1) to obtain global asymptotic stability results for those equations. The obtained results will give the regions of the parametric space where the unique positive equilibrium of (1) is globally asymptotically stable. In an upcoming manuscript we will give more precisely the dynamics in some special cases of (1) such as the case where the right-hand side of (1) is decreasing in $x_{n}$ and increasing in $x_{n-1}$; here the theory of monotone maps can be applied to give the global dynamics. The application of the monotone map theory requires precise information on the local stability of the equilibrium solutions and the period-two solutions which will be given in this paper. See $[20,21]$ for an application of the monotone maps techniques to some competitive systems of linear fractional difference equations. These results will give the parameter regions where a global period-doubling bifurcation takes place, see [9]. The special cases of (1) where the unique equilibrium changes its stability character from the local stability to repeller are cases where the Naimark-Sacker bifurcation occurs, see $[8,22]$, and these cases will be treated in an upcoming manuscript. Following the approach from [11], we divide (1) into 49 special cases of types $(k, m)$ where $k$ (resp. $m$ ) denotes the number of positive parameters in the numerator (resp. denominator). We summarize information as regards the stability of both the equilibrium solution and the period-two solution as well as the monotonic character of the right-hand side of the special cases of types $(1,2),(2,1),(1,3),(3,1)$ and $(2,2)$ of $(1)$ in Tables $1-5$. We did not include the cases of the type $(1,1)$, which are well known from [11] as well as 7 cases 
Table 1 Equations of type $(1,2)$

\begin{tabular}{|c|c|c|c|c|}
\hline Equation & Equilibrium point & $\begin{array}{l}\text { Stability of } \\
\text { equilibrium point }\end{array}$ & $\begin{array}{l}\text { Period-two } \\
\text { solution and } \\
\text { stability }\end{array}$ & $\begin{array}{l}\text { Partial } \\
\text { derivatives }\end{array}$ \\
\hline$x_{n+1}=\frac{\beta x_{n-1} x_{n}}{C x_{n-1}^{2}+x_{n}}$ & $\begin{array}{l}\bar{x}=\frac{\beta-1}{c} \text { for } \beta>1 \\
\text { no eq. point for } \beta \leq 1\end{array}$ & LAS for $\beta>1$ & $\begin{array}{l}\text { no period-two } \\
\text { solution }\end{array}$ & $\begin{aligned} f_{u}^{\prime} & =\frac{C v^{3} \beta}{\left(C v^{2}+u\right)^{2}} \\
f_{v}^{\prime} & =\frac{u \beta\left(u-C V^{2}\right)}{\left(C v^{2}+u\right)^{2}}\end{aligned}$ \\
\hline$x_{n+1}=\frac{\gamma x_{n-1}^{2}}{C x_{n-1}^{2}+x_{n}}$ & $\begin{array}{l}\bar{x}=\frac{\gamma-1}{C} \text { for } \gamma>1 \\
\text { no eq. point for } \\
\gamma \leq 1\end{array}$ & $\begin{array}{l}\text { LAS for } \gamma>3 \\
\text { a saddle point } \\
\text { for } 1<\gamma<3 \\
\text { a non-hyp. eq. } \\
\text { for } \gamma=3\end{array}$ & $\begin{array}{l}\{0, \gamma / C\} \text {-LAS } \\
\left\{\frac{\gamma+1-\sqrt{(\gamma-3)(\gamma+1)}}{2 C},\right. \\
\left.\frac{\gamma+1+\sqrt{(\gamma-3)(\gamma+1)}}{2 C}\right\} \\
\text { a saddle point for } \\
\gamma>3 \\
\text { a non-hyp. eq. for } \\
\gamma=3\end{array}$ & $\begin{aligned} f_{u}^{\prime} & =-\frac{\gamma v^{2}}{\left(C v^{2}+u\right)^{2}} \\
f_{v}^{\prime} & =\frac{2 \gamma u v}{\left(C v^{2}+u\right)^{2}}\end{aligned}$ \\
\hline$x_{n+1}=\frac{\gamma x_{n-1}^{2}}{B x_{n-1} x_{n}+x_{n}}$ & $\begin{array}{l}\bar{x}=\frac{\gamma-1}{B} \text { for } \gamma>1 \\
\text { no eq. point for } \\
\gamma \leq 1\end{array}$ & $\begin{array}{l}\text { a saddle point for } \\
\gamma>1\end{array}$ & $\begin{array}{l}\text { no period-two } \\
\text { solution }\end{array}$ & $\begin{array}{l}f_{u}^{\prime}=-\frac{\gamma v^{2}}{u^{2}(B v+1)} \\
f_{v}^{\prime}=\frac{\gamma v(B v+2)}{u(B v+1)^{2}}\end{array}$ \\
\hline$x_{n+1}=\frac{\delta x_{n}}{B x_{n} x_{n-1}+x_{n-1}^{2}}$ & $\bar{x}=\sqrt{\frac{\delta}{B+1}}$ & $\begin{array}{l}\text { a repeller for } \delta>0 \\
B>0\end{array}$ & $\begin{array}{l}\text { no period-two } \\
\text { solution }\end{array}$ & $\begin{array}{l}f_{u}^{\prime}=\frac{\delta}{(B u+v)^{2}} \\
f_{v}^{\prime}=-\frac{u \delta(B u+2 v)}{\left(B u+v^{2}\right)^{2}}\end{array}$ \\
\hline$x_{n+1}=\frac{\delta x_{n}}{C x_{n-1}^{2}+x_{n}}$ & $\bar{x}=\frac{\sqrt{4 C \delta+1}-1}{2 C}$ & $\begin{array}{l}\text { LAS for } c \delta<2 \\
\text { a repeller for } c \delta>2 \\
\text { a non-hyp. eq. for } \\
c \delta=2\end{array}$ & $\begin{array}{l}\text { no period-two } \\
\text { solution }\end{array}$ & $\begin{aligned} f_{u}^{\prime} & =\frac{C V^{2} \delta}{\left(C v^{2}+u\right)^{2}} \\
f_{v}^{\prime} & =-\frac{2 C u v \delta}{\left(C v^{2}+u\right)^{2}}\end{aligned}$ \\
\hline
\end{tabular}

of types $(3,2),(2,3)$, and $(3,3)$ for which global stability will be given in Section 6 . Using the techniques established in [23-25] one can determine the rate of convergence for all regions of parameters for which we established convergence.

Some special cases of (1) have very interesting dynamics such as $x_{n+1}=\frac{\beta x_{n-1} x_{n}}{C x_{n-1}^{2}+x_{n}}$ given in Table 1 where, in the case $\beta \leq 1$, every solution converges to 0 although 0 is out of range of this equation. Another interesting example is the equation $x_{n+1}=\frac{\gamma x_{n-1}^{2}}{C x_{n-1}^{2}+x_{n}}$ from Table 1, where, in the case $\gamma \leq 1$, every solution converges to 0 or to the unique periodtwo solution. It is interesting to notice that 0 is out of the range of this equation. Another interesting example is the equation $x_{n+1}=\frac{\beta x_{n} x_{n-1}+\gamma x_{n-1}^{2}}{x_{n}}$ from Table 2, which has the property that if $\beta \geq 1$ every solution approaches $\infty$. None of these dynamics scenarios were possible in the case of the linear fractional difference equation, which is also a special case of (2) and which was studied in great detail in [11].

\section{Local stability of the positive equilibrium}

In this section we investigate the equilibrium points of Eq. (1) where $\beta, \gamma, \delta, A, B, C \in$ $[0, \infty), \beta+\gamma+\delta, A+B+C \in(0, \infty)$ and where the initial conditions $x_{-1}$ and $x_{0}$ are arbitrary nonnegative real numbers such $B x_{n} x_{n-1}+C x_{n-1}^{2}+D x_{n}>0$ for all $n \geq 0$.

In view of the above restriction on the initial conditions of (1), the equilibrium points of (1) are positive solutions of the equation

$$
\bar{x}=\frac{\beta \bar{x}^{2}+\gamma \bar{x}^{2}+\delta \bar{x}}{B \bar{x}^{2}+C \bar{x}^{2}+D \bar{x}},
$$

or equivalently

$$
\bar{x}^{2}(B+C)+\bar{x}(D-\beta-\gamma)-\delta=0 .
$$


Table 2 Equations of type $(2,1)$

\begin{tabular}{|c|c|c|c|c|}
\hline Equation & Equilibrium point & $\begin{array}{l}\text { Stability of } \\
\text { equilibrium point }\end{array}$ & $\begin{array}{l}\text { Period-two } \\
\text { solution and } \\
\text { stability }\end{array}$ & $\begin{array}{l}\text { Partial } \\
\text { derivatives }\end{array}$ \\
\hline$x_{n+1}=\frac{\beta x_{n} x_{n-1}+\gamma x_{n-1}^{2}}{x_{n}}$ & $\begin{array}{l}\bar{x}=t, t>0 \text { for } \\
\beta+\gamma=1 \\
\text { no eq. point for } \\
\beta+\gamma \neq 1\end{array}$ & $\begin{array}{l}\text { a non-hyp. for } \\
\beta+\gamma=1\end{array}$ & $\begin{array}{l}\text { no minimal } \\
\text { period-two solution }\end{array}$ & $\begin{array}{l}f_{u}^{\prime}=-\frac{v^{2} \gamma}{u^{2}} \\
f_{v}^{\prime}=\frac{2 v \gamma}{u}+\beta\end{array}$ \\
\hline$x_{n+1}=\frac{\beta x_{n-1} x_{n}+\delta x_{n}}{x_{n-1}^{2}}$ & $\bar{x}=\frac{1}{2}\left(\sqrt{\beta^{2}+4 \delta}+\beta\right)$ & $\begin{array}{l}\text { a repeller for } \\
\beta, \delta>0\end{array}$ & $\begin{array}{l}\text { no minimal } \\
\text { period-two solution }\end{array}$ & $\begin{array}{l}f_{u}^{\prime}=\frac{v \beta+\delta}{v^{2}} \\
f_{v}^{\prime}=-\frac{u(v \beta+2 \delta)}{v^{3}}\end{array}$ \\
\hline$x_{n+1}=\frac{\gamma x_{n-1}^{2}+\delta x_{n}}{x_{n-1} x_{n}}$ & $\bar{x}=\frac{1}{2}\left(\sqrt{\gamma^{2}+4 \delta}+\gamma\right)$ & $\begin{array}{l}\text { LAS for } 4 \delta>3 \gamma^{2} \\
\text { a saddle point for } \\
4 \delta<3 \gamma^{2} \\
\text { a non-hyp. for } \\
4 \delta=3 \gamma^{2}\end{array}$ & $\begin{array}{l}\left\{\frac{\gamma \delta+\sqrt{\delta^{2}\left(4 \delta-3 \gamma^{2}\right)}}{2\left(\gamma^{2}-\delta\right)}\right. \\
\left.\frac{\gamma \delta-\sqrt{\delta^{2}\left(4 \delta-3 \gamma^{2}\right)}}{2\left(\gamma^{2}-\delta\right)}\right\} \\
\text { exists for } \\
3 \gamma^{2}<4 \delta<4 \gamma^{2} \\
\text { a saddle point } \\
\text { for } 3 \gamma^{2}<4 \delta<4 \gamma^{2}\end{array}$ & $\begin{array}{l}f_{u}^{\prime}=-\frac{v \gamma}{u^{2}} \\
f_{v}^{\prime}=\frac{v^{2} \gamma-u \delta}{u v^{2}}\end{array}$ \\
\hline$x_{n+1}=\frac{\gamma x_{n-1}^{2}+\delta x_{n}}{x_{n-1}^{2}}$ & $\bar{x}=\frac{1}{2}\left(\sqrt{\gamma^{2}+4 \delta}+\gamma\right)$ & $\begin{array}{l}\text { LAS for } \delta<2 \gamma^{2} \\
\text { a repeller for } \delta>2 \gamma^{2} \\
\text { a non-hyp. for } \\
\delta=2 \gamma^{2}\end{array}$ & $\begin{array}{l}\text { possible } \\
\text { Naimark-Sacker } \\
\text { bifurcation }\end{array}$ & $\begin{array}{l}f_{u}^{\prime}=\frac{\delta}{v^{2}} \\
f_{v}^{\prime}=\frac{-2 u \delta}{v^{3}}\end{array}$ \\
\hline$x_{n+1}=\frac{\gamma x_{n-1}^{2}+\delta x_{n}}{x_{n}}$ & $\bar{x}=\frac{\delta}{\gamma-1}$ for $\gamma<1$ & $\begin{array}{l}\text { LAS for } 3 \gamma<1 \\
\text { a saddle point for } \\
3 \gamma>1 \\
\text { a non-hyp. for } 3 \gamma=1\end{array}$ & $\begin{array}{l}\{\phi, \psi\} \text { for } 3 \gamma<1 \\
\phi=\frac{-\sqrt{(\gamma+1)(1-3 \gamma) \delta^{2}}+\gamma \delta+\delta}{2 \gamma(\gamma+1)} \\
\psi=\frac{\sqrt{(\gamma+1)(1-3 \gamma) \delta^{2}}+\gamma \delta+\delta}{2 \gamma(\gamma+1)} \\
\text { a saddle point for } 3 \gamma<1\end{array}$ & $\begin{array}{l}f_{u}^{\prime}=-\frac{v^{2} \gamma}{u^{2}} \\
f_{v}^{\prime}=\frac{2 v \gamma}{u}\end{array}$ \\
\hline
\end{tabular}

When

$$
\delta=0, \quad B+C>0 \text { and } \beta+\gamma>D
$$

the unique positive equilibrium of (1) is given by

$$
\bar{x}=\frac{\beta+\gamma-D}{B+C} \text {. }
$$

When

$$
\delta>0, \quad B+C=0 \text { and } \quad D>\beta+\gamma
$$

the unique positive equilibrium of (1) is given by

$$
\bar{x}=\frac{\delta}{D-\beta-\gamma} .
$$

Finally when

$$
\delta>0 \text { and } B+C>0
$$

the only equilibrium point of (1) is the positive solution

$$
\bar{x}=\frac{\sqrt{4 \delta(B+C)+(D-\beta-\gamma)^{2}}-D+\beta+\gamma}{2(B+C)}
$$

of the quadratic equation (8). 
Table 3 Equations of type $(2,2)$

\begin{tabular}{|c|c|c|c|c|}
\hline Equation & Equilibrium point & $\begin{array}{l}\text { Stability of } \\
\text { equilibrium point }\end{array}$ & $\begin{array}{l}\text { Period-two } \\
\text { solution and } \\
\text { stability }\end{array}$ & $\begin{array}{l}\text { Partial } \\
\text { derivatives }\end{array}$ \\
\hline$x_{n+1}=\frac{\beta x_{n} x_{n-1}+\gamma x_{n-1}^{2}}{C x_{n-1}^{2}+x_{n}}$ & $\begin{array}{l}\bar{x}=\frac{\beta+\gamma-1}{C} \text { for } \\
\beta+\gamma>1 \\
\text { no eq. point for } \\
\beta+\gamma \leq 1\end{array}$ & $\begin{array}{l}\text { LAS for }(3 \beta+\gamma> \\
3 \wedge \gamma \leq 3) \vee \gamma>3 \\
\text { a saddle point for } \\
\beta<1 \wedge \beta+\gamma> \\
1 \wedge 3 \beta+\gamma<3 \\
\text { a non-hyp. eq. for } \\
\gamma<3 \wedge 3 \beta+\gamma=3\end{array}$ & $\begin{array}{l}\left\{\phi_{1}, \psi_{1}\right\}=\{0, \gamma / C\} \text {-LAS for } \\
\beta<1 \\
\text { saddle for } \beta>1 \\
\text { non-hyp. for } \beta=1 \\
\left\{\phi_{2}, \psi_{2}\right\} \text { exists for } \beta<1 \\
3 \beta+\gamma>3 \\
\text { a saddle point for } \beta<1, \\
3 \beta+\gamma>3 \\
\psi_{1}= \\
\frac{(\gamma+1-\beta)+\sqrt{(\gamma+1-\beta)(3 \beta+\gamma-3)}}{2 C} \\
\phi_{2}= \\
\frac{(\gamma+1-\beta)-\sqrt{(\gamma+1-\beta)(3 \beta+\gamma-3)}}{2 c}\end{array}$ & $\begin{array}{l}f_{u}^{\prime}=\frac{v^{2}(C v \beta-\gamma)}{\left(C v^{2}+u\right)^{2}} \\
f_{v}^{\prime}=\frac{u(v(2 \gamma-C v \beta)+u \beta)}{\left(C v^{2}+u\right)^{2}}\end{array}$ \\
\hline$x_{n+1}=\frac{\beta x_{n} x_{n-1}+\gamma x_{n-1}^{2}}{B x_{n-1} x_{n}+x_{n}}$ & $\begin{array}{l}\bar{x}=\frac{\beta+\gamma-1}{B} \text { for } \\
\beta+\gamma>1\end{array}$ & $\begin{array}{l}\text { LAS for } \beta>\gamma+1 \\
\text { a saddle for } \\
1-\gamma<\beta<\gamma+1 \\
\text { a non-hyp. for } \\
\beta=\gamma+1\end{array}$ & $\begin{array}{l}\{\phi, \psi\} \text { for } \phi, \psi>0 \\
\text { for } \beta=\gamma+1\end{array}$ & $\begin{array}{l}f_{u}^{\prime}=-\frac{v^{2} \gamma}{u^{2}(B v+1)} \\
f_{v}^{\prime}=\frac{v \gamma(B v+2)+u \beta}{u(B v+1)^{2}}\end{array}$ \\
\hline$x_{n+1}=\frac{\beta x_{n-1} x_{n}+\delta x_{n}}{B x_{n} x_{n-1}+x_{n-1}^{2}}$ & $\bar{x}=\frac{\sqrt{4 B \delta+\beta^{2}+4 \delta}+\beta}{2(B+1)}$ & $\begin{array}{l}\text { LAS for } B \beta^{2}>\delta \\
\text { a repeller for } B \beta^{2}<\delta \\
\text { a non-hyp. for } \\
B \beta^{2}=\delta\end{array}$ & $\begin{array}{l}\text { no minimal } \\
\text { period-two solution }\end{array}$ & $\begin{array}{l}f_{u}^{\prime}=\frac{v \beta+\delta}{(B u+v)^{2}} \\
f_{v}^{\prime}=\frac{-u\left(B u \delta+v^{2} \beta+2 v \delta\right)}{v^{2}(B u+v)^{2}}\end{array}$ \\
\hline$x_{n+1}=\frac{\beta x_{n-1} x_{n}+\delta x_{n}}{C x_{n-1}^{2}+x_{n}}$ & $\bar{x}=\frac{\sqrt{4 C \delta+(\beta-1)^{2}}+\beta-1}{2 C}$ & $\begin{array}{l}\text { a LAS for } \\
c \delta<2 D(1+\beta) \\
\text { a repeller for } \\
c \delta>2 D(1+\beta) \\
\text { a non-hyp. eq. for } \\
c \delta=2 D(1+\beta)\end{array}$ & $\begin{array}{l}\text { no minimal } \\
\text { period-two solution }\end{array}$ & $\begin{array}{l}f_{u}^{\prime}=\frac{C v^{2}(v \beta+\delta)}{\left(\left(v^{2}+u\right)^{2}\right.} \\
f_{v}^{\prime}=\frac{u(u \beta-C v(v \beta+2 \delta))}{\left(C v^{2}+u\right)^{2}}\end{array}$ \\
\hline$x_{n+1}=\frac{\gamma x_{n-1}^{2}+\delta x_{n}}{B x_{n-1} x_{n}+x_{n-1}^{2}}$ & $\bar{x}=\frac{\sqrt{4 B \delta+\gamma^{2}+4 \delta}+\gamma}{2(B+1)}$ & $\begin{array}{l}\text { LAS for } \frac{3(B-1) \gamma^{2}}{(B+2)^{2}}<4 \delta \\
\text { or } \delta<2(2 B+1) \gamma^{2} \\
\text { saddle point for } \\
B>1 \wedge \delta<\frac{3(B-1) \gamma^{2}}{4(B+2)^{2}} \\
\text { non-hyp. eq. for } \\
(4 b+2) \gamma^{2}=\delta \text { or } \\
4(B+2)^{2} \delta=3(B-1) \gamma^{2} \\
\text { repeller for } \\
\delta>2(2 B+1) \gamma^{2}\end{array}$ & $\begin{array}{l}\text { possible } \\
\text { Naimark-Sacker } \\
\text { bifurcation }\end{array}$ & $\begin{array}{l}f_{u}^{\prime}=\frac{\delta-B v \gamma}{(B u+v)^{2}} \\
f_{v}^{\prime}=\frac{u\left(B v^{2} \gamma-2 v \delta-B u \delta\right)}{v^{2}(B u+v)^{2}}\end{array}$ \\
\hline$x_{n+1}=\frac{\gamma x_{n-1}^{2}+\delta x_{n}}{B x_{n} x_{n-1}+x_{n}}$ & $\bar{x}=\frac{\sqrt{4 B \delta+(1-\gamma)^{2}}+\gamma-1}{2 B}$ & $\begin{array}{l}\text { LAS for } \delta>\frac{(\gamma+1)(3 \gamma-1)}{4 B} \\
\text { a saddle point for } \\
\delta<\frac{(\gamma+1)(3 \gamma-1)}{4 B} \\
\text { a non-hyperbolic eq. } \\
\text { for } \delta=\frac{(\gamma+1)(3 \gamma-1)}{4 B}\end{array}$ & $\begin{array}{l}\{\phi, \psi\} \text { exists for } \\
\frac{(\gamma+1)(3 \gamma-1)}{4 B}<\delta<\frac{\gamma(\gamma+1)}{B} \\
\phi= \\
\frac{\delta(-\sqrt{4 B \delta-\gamma(3 \gamma+2)+1}+\gamma+1)}{2\left(-B \delta+\gamma^{2}+\gamma\right)} \\
\psi= \\
\frac{\delta(\sqrt{4 B \delta-\gamma(3 \gamma+2)+1}+\gamma+1)}{2\left(-B \delta+\gamma^{2}+\gamma\right)} \\
\text { a saddle point for } \\
\frac{(\gamma+1)(3 \gamma-1)}{4 B}<\delta<\frac{\gamma(\gamma+1)}{B}\end{array}$ & $\begin{array}{l}f_{u}^{\prime}=-\frac{v^{2} \gamma}{u^{2}(B v+1)} \\
f_{v}^{\prime}=\frac{v \gamma(B v+2)-B u \delta}{u(B v+1)^{2}}\end{array}$ \\
\hline$x_{n+1}=\frac{\gamma x_{n-1}^{2}+\delta x_{n}}{C x_{n-1}^{2}+x_{n}}$ & $\bar{x}=\frac{\sqrt{4 C \delta+(1-\gamma)^{2}}+\gamma-1}{2 C}$ & $\begin{array}{l}\text { LAS } \delta<\frac{(\gamma+2)(2 \gamma+1)}{C} \\
\text { and } \frac{(3-\gamma)(3 \gamma-1)}{16 C}<\delta \\
\text { a repeller for } \\
\delta>\frac{(\gamma+2)(2 \gamma+1)}{C} \\
\text { a saddle for } \\
\frac{(3-\gamma)(3 \gamma-1)}{16 C}>\delta \\
\text { a non-hyp. eq. for } \\
\frac{(3-\gamma)(3 \gamma-1)}{16 C}=\delta \text { or } \\
\delta=\frac{(\gamma+2)(2 \gamma+1)}{\delta}\end{array}$ & $\begin{array}{l}\text { possible } \\
\text { Naimark-Sacker } \\
\text { bifurcation }\end{array}$ & $\begin{array}{l}f_{u}^{\prime}=\frac{v^{2}(C \delta-\gamma)}{\left(C v^{2}+u\right)^{2}} \\
f_{v}^{\prime}=\frac{2 u v(\gamma-C \delta)}{\left(C v^{2}+u\right)^{2}}\end{array}$ \\
\hline
\end{tabular}


Table 4 Equations of type $(3,1)$

\begin{tabular}{|c|c|c|c|c|}
\hline Equation & Equilibrium point & $\begin{array}{l}\text { Stability of } \\
\text { equilibrium point }\end{array}$ & $\begin{array}{l}\text { Period-two } \\
\text { solution and } \\
\text { stability }\end{array}$ & $\begin{array}{l}\text { Partial } \\
\text { derivatives }\end{array}$ \\
\hline$x_{n+1}=\frac{\beta x_{n} x_{n-1}+\gamma x_{n-1}^{2}+\delta x_{n}}{x_{n-1} x_{n}}$ & $\bar{x}=\frac{\sqrt{(\beta+\gamma)^{2}+4 \delta}+\beta+\gamma}{2}$ & $\begin{array}{l}\text { LAS for } \beta>\gamma \text { or } \\
\beta \leq \gamma \wedge \beta^{2}+2 \beta \gamma+ \\
4 \delta>3 \gamma^{2} \\
\text { a saddle point for } \\
\beta<\gamma \wedge \beta^{2}+2 \beta \gamma+ \\
4 \delta<3 \gamma^{2} \\
\text { a non-hyp. eq. for } \\
\beta<\gamma \wedge \beta^{2}+2 \beta \gamma+ \\
4 \delta=3 \gamma^{2}\end{array}$ & $\begin{array}{l}\text { no minimal } \\
\text { period-two sol. }\end{array}$ & $\begin{array}{l}f_{u}^{\prime}=-\frac{v \gamma}{u^{2}} \\
f_{v}^{\prime}=\frac{v^{2} \gamma-u \delta}{u v^{2}}\end{array}$ \\
\hline$x_{n+1}=\frac{\beta x_{n} x_{n-1}+\gamma x_{n-1}^{2}+\delta x_{n}}{x_{n-1}^{2}}$ & $\bar{x}=\frac{\sqrt{(\beta+\gamma)^{2}+4 \delta}+\beta+\gamma}{2}$ & $\begin{array}{l}\text { LAS for } \delta<\gamma(\beta+2 \gamma) \\
\text { a repeller for } \\
\delta>\gamma(\beta+2 \gamma) \\
\text { a non-hyp. eq. for } \\
\delta=\gamma(\beta+2 \gamma)\end{array}$ & $\begin{array}{l}\text { possible } \\
\text { Naimark-Sacker } \\
\text { bifurcation }\end{array}$ & $\begin{array}{l}f_{u}^{\prime}=\frac{v \beta+\delta}{v^{2}} \\
f_{v}^{\prime}=\frac{-u v \beta-2 u \delta}{v^{3}}\end{array}$ \\
\hline$x_{n+1}=\frac{\beta x_{n} x_{n-1}+\gamma x_{n-1}^{2}+\delta x_{n}}{x_{n}}$ & $\bar{x}=\frac{\delta}{1-\beta-\gamma}$ & $\begin{array}{l}\text { LAS for } \beta>\gamma \text { or } \\
\beta+3 \gamma<1 \\
\text { a saddle for } \\
\beta+3 \gamma>1 \\
\text { a non-hyperbolic eq. } \\
\text { for } \beta+3 \gamma=1\end{array}$ & $\begin{array}{l}\{\phi, \psi\} \text { exists for } \\
\beta+3 \gamma<1 \\
\phi= \\
\frac{\delta(\gamma-\beta+1+\sqrt{(\beta-\gamma-1)(\beta+3 \gamma-1))}}{2 \gamma(-\beta+\gamma+1)} \\
\psi= \\
\frac{\delta(\gamma-\beta+1-\sqrt{(\beta-\gamma-1)(\beta+3 \gamma-1))}}{2 \gamma(-\beta+\gamma+1)} \\
\text { a saddle point for } \\
\beta+3 \gamma<1\end{array}$ & $\begin{array}{l}f_{u}^{\prime}=-\frac{v^{2} \gamma}{u^{2}} \\
f_{v}^{\prime}=\frac{u \beta+2 v \gamma}{u}\end{array}$ \\
\hline
\end{tabular}

Table 5 Equations of type $(1,3)$

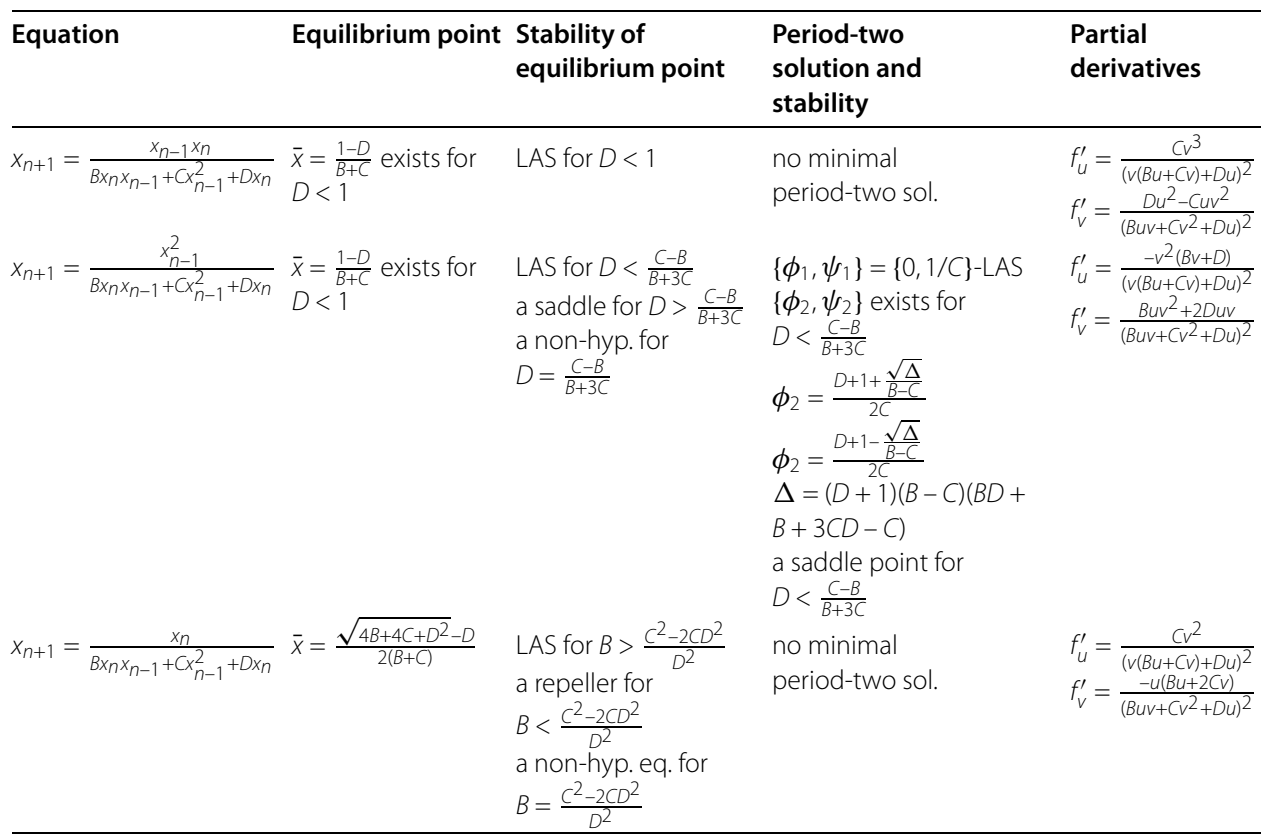


In summary, it is interesting to observe that when (1) has a positive equilibrium $\bar{x}$, then $\bar{x}$ is unique and it satisfies (7) and (8). This observation simplifies the investigation of the local stability of the positive equilibrium of (1).

Next, we investigate the stability of the positive equilibrium of (1). Set

$$
f(u, v)=\frac{u v \beta+u \delta+v^{2} \gamma}{B u v+C v^{2}+D u}
$$

and observe that

$$
f_{u}(u, v)=\frac{v^{2}(C(v \beta+\delta)-\gamma(B v+D))}{(v(B u+C v)+D u)^{2}}
$$

and

$$
f_{v}(u, v)=\frac{u\left(-B u \delta+B v^{2} \gamma-C v(v \beta+2 \delta)+D(u \beta+2 v \gamma)\right)}{(v(B u+C v)+D u)^{2}}
$$

If $\bar{x}$ denotes an equilibrium point of (1), then the linearized equation associated with (1) about the equilibrium point $\bar{x}$ is

$$
z_{n+1}=p z_{n}+q z_{n-1}
$$

where

$$
p=f_{u}(\bar{x}, \bar{x}) \quad \text { and } \quad q=f_{v}(\bar{x}, \bar{x}) .
$$

\section{Theorem 3 Assume that}

$$
\delta=0, \quad B+C>0 \text { and } \beta+\gamma>D .
$$

\section{Then the unique equilibrium point}

$$
\bar{x}=\frac{\beta+\gamma-D}{B+C}
$$

of (1) is

(i) locally asymptotically stable if $C(-3 D+3 \beta+\gamma)>B(D-\beta+\gamma)$;

(ii) a saddle point if $C(-3 D+3 \beta+\gamma)<B(D-\beta+\gamma)$;

(iii) a non-hyperbolic equilibrium if $C(-3 D+3 \beta+\gamma)=B(D-\beta+\gamma)$ or

$$
(B=\gamma=D=0 \wedge \beta>0 \wedge C>0) \text {. }
$$

Proof It is easy to see that

$$
p=f_{u}(\bar{x}, \bar{x})=\frac{C(\beta-D)-B \gamma}{(B+C)(\beta+\gamma)} \quad \text { and } \quad q=f_{v}(\bar{x}, \bar{x})=\frac{B(D+\gamma)+C(2 D-\beta)}{(B+C)(\beta+\gamma)} \text {. }
$$

Then the proof follows from Theorem 1.1.1 in [11] and the fact that

$$
1-p-q=\frac{\beta+\gamma-D}{\beta+\gamma}>0, \quad p-q+1=\frac{C(-3 D+3 \beta+\gamma)-B(D-\beta+\gamma)}{(B+C)(\beta+\gamma)}
$$


and

$$
q+1=\frac{B(D+\beta+2 \gamma)+C(2 D+\gamma)}{(B+C)(\beta+\gamma)} \geq 0
$$

Theorem 4 Assume that

$$
\delta>0, \quad B+C=0 \text { and } D>\beta+\gamma .
$$

Then the unique equilibrium point

$$
\bar{x}=\frac{\delta}{D-\beta-\gamma}
$$

of (1) is

(i) locally asymptotically stable if $D>\beta+3 \gamma$;

(ii) a saddle point if $\beta+\gamma<D<\beta+3 \gamma$;

(iii) a non-hyperbolic if $D=\beta+3 \gamma$.

Proof It is easy to see that

$$
p=f_{u}(\bar{x}, \bar{x})=-\frac{\gamma}{D} \quad \text { and } \quad q=f_{v}(\bar{x}, \bar{x})=\frac{\beta+2 \gamma}{D}
$$

Then the proof follows from Theorem 1.1.1 in [11] and the fact that

$$
1-p-q=\frac{D-\beta-\gamma}{\beta+\gamma}, \quad p-q+1=\frac{D-\beta-3 \gamma}{D}, \quad q+1=\frac{D+\beta+2 \gamma}{D} \text {. }
$$

As we previously mentioned if

$$
\delta>0 \text { and } B+C>0
$$

the only equilibrium point of (1) is the positive solution

$$
\bar{x}=\frac{\sqrt{4 \delta(B+C)+(D-\beta-\gamma)^{2}}-D+\beta+\gamma}{2(B+C)}
$$

of the quadratic equation (8).

By using the identity

$$
\bar{x}^{2}(B+C)=\bar{x}(\beta+\gamma-D)+\delta
$$

one can see that

$$
\begin{aligned}
p & =f_{u}(\bar{x}, \bar{x})=\frac{\bar{x}(C \beta-B \gamma)+C \delta-D \gamma}{(\bar{x}(B+C)+D)^{2}} \\
& =\frac{\bar{x}(C \beta-B \gamma)+C \delta-D \gamma}{2 D \bar{x}(B+C)+\bar{x}^{2}(B+C)^{2}+D^{2}} \\
& =\frac{\bar{x}(C \beta-B \gamma)+C \delta-D \gamma}{\bar{x}(B+C)(D+\beta+\gamma)+\delta(B+C)+D^{2}},
\end{aligned}
$$




$$
\begin{aligned}
q & =f_{v}(\bar{x}, \bar{x})=\frac{\bar{x}(B \gamma-C \beta)-\delta(B+2 C)+D(\beta+2 \gamma)}{(\bar{x}(B+C)+D)^{2}} \\
& =\frac{\bar{x}(B \gamma-C \beta)-\delta(B+2 C)+D(\beta+2 \gamma)}{2 D \bar{x}(B+C)+\bar{x}^{2}(B+C)^{2}+D^{2}} \\
& =\frac{\bar{x}(B \gamma-C \beta)-\delta(B+2 C)+D(\beta+2 \gamma)}{\bar{x}(B+C)(D+\beta+\gamma)+\delta(B+C)+D^{2}},
\end{aligned}
$$

and

$$
\begin{aligned}
& p-q+1=\frac{\bar{x}(B(D+\beta-\gamma)+C(D+3 \beta+\gamma))+2 \delta(B+2 C)+D^{2}-D(\beta+3 \gamma)}{\bar{x}(B+C)(D+\beta+\gamma)+\delta(B+C)+D^{2}}, \\
& 1-p-q=\frac{\bar{x}(B+C)(D+\beta+\gamma)+2 \delta(B+C)+D^{2}-D(\beta+\gamma)}{\bar{x}(B+C)(D+\beta+\gamma)+\delta(B+C)+D^{2}}, \\
& q+1=\frac{\bar{x}(B(D+\beta+2 \gamma)+C(D+\gamma))-C \delta+D(D+\beta+2 \gamma)}{\bar{x}(B+C)(D+\beta+\gamma)+\delta(B+C)+D^{2}} \\
& q-1=\frac{\bar{x}(B \gamma-C \beta)-\delta(B+2 C)+D(\beta+2 \gamma)}{\bar{x}(B+C)(D+\beta+\gamma)+\delta(B+C)+D^{2}} .
\end{aligned}
$$

Let

$$
\begin{aligned}
& \rho_{1}=\frac{D(-D+\beta+3 \gamma)-2 \delta(B+2 C)}{B(D+\beta-\gamma)+C(D+3 \beta+\gamma)}, \\
& \rho_{2}=\frac{D(-D+\beta+\gamma)-2 \delta(B+C)}{(B+C)(D+\beta+\gamma)}, \\
& \rho_{3}=\frac{C \delta-D(D+\beta+2 \gamma)}{B(D+\beta+2 \gamma)+C(D+\gamma)} .
\end{aligned}
$$

Now if we set

$$
F(u)=u^{2}(B+C)+u(D-\beta-\gamma)-\delta
$$

it is clear that $F(\bar{x})=0$ and that $\bar{x}>\rho$ if and only if $F(\rho)<0$ while $\bar{x}<\sigma$ if and only if $F(\sigma)>0$ for some $\rho, \sigma \in[0, \infty)$.

A straightforward computation gives

$$
\begin{aligned}
F\left(\rho_{1}\right)= & (\delta(B+C)-D(\beta+\gamma)) \\
& \times \frac{\left((D-\beta-3 \gamma)((B+3 C)(D-\beta)+\gamma(B-C))+4 \delta(B+2 C)^{2}\right)}{(B D+B \beta-B \gamma+C D+3 C \beta+C \gamma)^{2}}, \\
F\left(\rho_{2}\right)= & \frac{(\delta(B+C)-D(\beta+\gamma))\left(4 \delta(B+C)+(-D+\beta+\gamma)^{2}\right)}{(B+C)(D+\beta+\gamma)^{2}}, \\
F\left(\rho_{3}\right)= & \frac{(D(\beta+\gamma)-\delta(B+C))\left(B(D+\beta+2 \gamma)^{2}+C((2 D+\gamma)(D+\beta+2 \gamma)-C \delta)\right)}{(B D+B \beta+2 B \gamma+C D+C \gamma)^{2}} .
\end{aligned}
$$

Lemma 1 Let $p$ and $q$ be partial derivatives given by (9) and (10). Assume that

$\delta>0, \quad B+C>0$. 
(a) Then $1-p-q>0$ is true for all values of parameters.

(b) Then $p-q+1>0$ if and only if

$$
\delta>-\frac{(D-\beta-3 \gamma)((B+3 C)(D-\beta)+\gamma(B-C))}{4(B+2 C)^{2}} .
$$

(c) Then $q+1>0$ if and only if

$$
C=0
$$

or

$$
C>0 \quad \text { and } \quad \delta<\frac{(D+\beta+2 \gamma)(B(D+\beta+2 \gamma)+C(2 D+\gamma))}{C^{2}} .
$$

Proof (a) The inequality $1-q-p>0$ is equivalent to

$$
D(-D+\beta+\gamma)-2 \delta(B+C) \leq 0 \quad \text { or } \quad\left(D(-D+\beta+\gamma)-2 \delta(B+C)>0 \text { and } F\left(\rho_{2}\right)<0\right) \text {, }
$$

which is equivalent to

$$
\begin{aligned}
& C \geq \frac{D(-D+\beta+\gamma)-2 B \delta}{2 \delta} \text { or } \\
& \left(C<\frac{D(-D+\beta+\gamma)-2 B \delta}{2 \delta} \text { and } C<\frac{-B \delta+D \beta+D \gamma}{\delta}\right) .
\end{aligned}
$$

Since

$$
\frac{-B \delta+D \beta+D \gamma}{\delta}-\frac{D(-D+\beta+\gamma)-2 B \delta}{2 \delta}=\frac{D^{2}+D \beta+D \gamma}{2 \delta} \geq 0
$$

we find that $1-q-p>0$ is always true.

(b) There are three cases to consider.

(i) Assume $B(D+\beta-\gamma)+C(D+3 \beta+\gamma)>0$. Then $p-q+1>0$ is equivalent to $\bar{x}>\rho_{1}$. One can see that

$$
F\left(\rho_{1}\right)<0
$$

if and only if

$$
\delta \in\left(-\frac{(D-\beta-3 \gamma)((B+3 C)(D-\beta)+\gamma(B-C))}{4(B+2 C)^{2}}, \frac{D(\beta+\gamma)}{B+C}\right),
$$

since

$$
\begin{aligned}
& \frac{D(\beta+\gamma)}{B+C}+\frac{(D-\beta-3 \gamma)((B+3 C)(D-\beta)+\gamma(B-C))}{4(B+2 C)^{2}} \\
& \quad=\frac{(B(D+\beta-\gamma)+C(D+3 \beta+\gamma))(B(D+\beta+3 \gamma)+C(3 D+\beta+3 \gamma))}{4(B+C)(B+2 C)^{2}}>0 .
\end{aligned}
$$


From (12), we have $p-q+1>0$ if and only if

$$
\delta \geq-\frac{D(D-\beta-3 \gamma)}{2(B+2 C)} \quad \text { or } \quad\left(\delta<-\frac{D(D-\beta-3 \gamma)}{2(B+2 C)} \text { and } F\left(\rho_{1}\right)<0\right) \text {, }
$$

which is equivalent to

$$
\begin{aligned}
& \delta \geq-\frac{D(D-\beta-3 \gamma)}{2(B+2 C)} \text { or } \\
& \delta \in\left(-\frac{(D-\beta-3 \gamma)((B+3 C)(D-\beta)+\gamma(B-C))}{4(B+2 C)^{2}},-\frac{D(D-\beta-3 \gamma)}{2(B+2 C)}\right),
\end{aligned}
$$

since

$$
\begin{gathered}
\frac{D(D-\beta-3 \gamma)}{2(B+2 C)}-\frac{(D-\beta-3 \gamma)((B+3 C)(D-\beta)+\gamma(B-C))}{4(B+2 C)^{2}} \\
=\frac{(D-\beta-3 \gamma)(B(D+\beta-\gamma)+C(D+3 \beta+\gamma))}{4(B+2 C)^{2}}<0
\end{gathered}
$$

and

$$
\frac{D(D-\beta-3 \gamma)}{2(B+2 C)}+\frac{D(\beta+\gamma)}{B+C}=\frac{D(B(D+\beta-\gamma)+C(D+3 \beta+\gamma))}{2(B+C)(B+2 C)} .
$$

Statement (13) is equivalent to

$$
\delta>-\frac{(D-\beta-3 \gamma)((B+3 C)(D-\beta)+\gamma(B-C))}{4(B+2 C)^{2}},
$$

from which the proof follows.

(ii) Assume $B(D+\beta-\gamma)+C(D+3 \beta+\gamma)<0$. Then $p-q+1>0$ if and only if $\bar{x}<\rho_{1}$. It is easy to see that

$$
F\left(\rho_{1}\right)>0
$$

if and only if

$$
\delta<\frac{D(\beta+\gamma)}{B+C} \quad \text { or } \quad \delta>-\frac{(D-\beta-3 \gamma)((B+3 C)(D-\beta)+\gamma(B-C))}{4(B+2 C)^{2}},
$$

which implies that $p-q+1>0$ if and only if

$$
\delta>-\frac{D(D-\beta-3 \gamma)}{2(B+2 C)} \text { and } F\left(\rho_{1}\right)>0
$$

which is equivalent to

$$
\delta>-\frac{D(D-\beta-3 \gamma)}{2(B+2 C)} \text { and } \delta>-\frac{(D-\beta-3 \gamma)((B+3 C)(D-\beta)+\gamma(B-C))}{4(B+2 C)^{2}} \text {, }
$$

since

$$
\frac{D(D-\beta-3 \gamma)}{2(B+2 C)}+\frac{D(\beta+\gamma)}{B+C}=\frac{D(B(D+\beta-\gamma)+C(D+3 \beta+\gamma))}{2(B+C)(B+2 C)}<0 .
$$


In view of the left-hand side of (14) we see that (15) is equivalent to

$$
\delta>-\frac{(D-\beta-3 \gamma)((B+3 C)(D-\beta)+\gamma(B-C))}{4(B+2 C)^{2}},
$$

from which the proof follows.

(iii) If $B(D+\beta-\gamma)+C(D+3 \beta+\gamma)=0$, then the proof follows from (11).

(c) The inequality $q>-1$ is equivalent to

$$
C \delta-D(D+\beta+2 \gamma) \leq 0 \quad \text { or } \quad\left(C \delta-D(D+\beta+2 \gamma)>0 \text { and } F\left(\rho_{3}\right)<0\right) \text {, }
$$

which is true for $C=0$. If $C>0$, then (16) is equivalent to

$$
\delta \leq \frac{D(D+\beta+2 \gamma)}{C}
$$

or

$$
\begin{aligned}
& \delta>\frac{D(D+\beta+2 \gamma)}{C} \text { and } \\
& \delta \in\left(\frac{D(\beta+\gamma)}{B+C}, \frac{(D+\beta+2 \gamma)(B(D+\beta+2 \gamma)+C(2 D+\gamma))}{C^{2}}\right)
\end{aligned}
$$

since

$$
\begin{aligned}
& \frac{(D+\beta+2 \gamma)(B(D+\beta+2 \gamma)+C(2 D+\gamma))}{C^{2}}-\frac{D(\beta+\gamma)}{B+C} \\
& =\frac{(B(D+\beta+2 \gamma)+C(D+\gamma))(B(D+\beta+2 \gamma)+C(2 D+\beta+2 \gamma))}{C^{2}(B+C)}>0 .
\end{aligned}
$$

It is easy to see that

$$
\begin{gathered}
\frac{D(D+\beta+2 \gamma)}{C}-\frac{(D+\beta+2 \gamma)(B(D+\beta+2 \gamma)+C(2 D+\gamma))}{C^{2}} \\
=-\frac{(D+\beta+2 \gamma)(B(D+\beta+2 \gamma)+C(D+\gamma))}{C^{2}} \leq 0
\end{gathered}
$$

and

$$
\frac{D(\beta+\gamma)}{B+C}-\frac{D(D+\beta+2 \gamma)}{C}=-\frac{D(B(D+\beta+2 \gamma)+C(D+\gamma))}{C(B+C)} \leq 0,
$$

from which it follows that (18) is equivalent to

$$
\delta \in\left(\frac{D(D+\beta+2 \gamma)}{C}, \frac{(D+\beta+2 \gamma)(B(D+\beta+2 \gamma)+C(2 D+\gamma))}{C^{2}}\right) .
$$

Since $C>0$, in view of (19) we find that (17) and (18) are equivalent to

$$
\delta<\frac{(D+\beta+2 \gamma)(B(D+\beta+2 \gamma)+C(2 D+\gamma))}{C^{2}} .
$$


Theorem 5 Assume

$$
\delta>0, \quad B+C>0 .
$$

Then the unique equilibrium point

$$
\bar{x}=\frac{\sqrt{4 \delta(B+C)+(D-\beta-\gamma)^{2}}-D+\beta+\gamma}{2(B+C)}
$$

of (1) is

(i) locally asymptotically stable if and only if any of the following holds:

(a)

$$
C>0
$$

and

$$
\begin{aligned}
&- \frac{(D-\beta-3 \gamma)((B+3 C)(D-\beta)+\gamma(B-C))}{4(B+2 C)^{2}} \\
&<\delta<\frac{(D+\beta+2 \gamma)(B(D+\beta+2 \gamma)+C(2 D+\gamma))}{C^{2}}
\end{aligned}
$$

(b)

$$
C=0 \quad \text { and } \quad \delta>-\frac{(D-\beta-3 \gamma)(D-\beta+\gamma)}{4 B}
$$

(ii) a repeller if and only if the following holds:

$$
C>0 \quad \text { and } \quad \delta>\frac{(D+\beta+2 \gamma)(B(D+\beta+2 \gamma)+C(2 D+\gamma))}{C^{2}}
$$

(iii) a saddle point if and only if the following holds:

$$
\delta<-\frac{(D-\beta-3 \gamma)((B+3 C)(D-\beta)+\gamma(B-C))}{4(B+2 C)^{2}} ;
$$

(iv) a non-hyperbolic equilibrium if and only if any of the following holds:

(a)

$$
\delta=-\frac{(D-\beta-3 \gamma)((B+3 C)(D-\beta)+\gamma(B-C))}{4(B+2 C)^{2}}
$$

(b)

$$
C>0 \quad \text { and } \quad \delta=\frac{(D+\beta+2 \gamma)(B(D+\beta+2 \gamma)+C(2 D+\gamma))}{C^{2}} \text {. }
$$

Proof The proof follows from Theorem 1.1.1 in [11] and Lemma 1. 


\section{Existence of period-two solutions}

Assume that $\{\phi, \psi\}$ is a minimal period-two solution of (1). Then

$$
\phi=f(\psi, \phi) \text { and } \quad \psi=f(\phi, \psi) \quad \text { with } \psi, \phi \in[0, \infty) \text { and } \phi \neq \psi \text {, }
$$

which is equivalent to

$$
\phi=\frac{\beta \phi \psi+\gamma \phi^{2}+\delta \psi}{B \phi \psi+C \phi^{2}+D \psi} \quad \text { and } \quad \psi=\frac{\beta \phi \psi+\gamma \psi^{2}+\delta \phi}{B \phi \psi+C \psi^{2}+D \phi},
$$

from which it immediately follows that

$$
\phi\left(B \phi \psi+C \phi^{2}+D \psi\right)=\beta \phi \psi+\gamma \phi^{2}+\delta \psi
$$

and

$$
\psi\left(B \phi \psi+C \psi^{2}+D \phi\right)=\beta \phi \psi+\gamma \psi^{2}+\delta \phi .
$$

Lemma 2 Equation (1) has a minimal period-two solution $\{\phi, \psi\}$ with $\phi \psi=0$ if and only if the following holds:

(i) $\delta=0, \gamma>0$ and $C>0$, then $\{\phi, \psi\}=\{0, \gamma / C\}$ is the minimal period-two solution.

(ii) $\delta=0, \gamma=0$ and $C=0$, then $\{\phi, \psi\}=\{0, \psi\}$, with $\psi \neq 0$ is a minimal period-two solution.

Proof If $\phi=0$, then (20) and (21) are equivalent to

$$
\psi \delta=0 \quad \text { and } \quad \psi^{2}(C \psi-\gamma)=0
$$

from which the proof follows.

Assume that $\phi \psi \neq 0$. Subtracting equations (20) and (21) we get

$$
(\phi-\psi)\left(\phi \psi(B+C)+C\left(\phi^{2}+\psi^{2}\right)-\gamma(\phi+\psi)+\delta\right)=0 .
$$

Dividing (20) by $\phi$ and (20) by $\psi$ and subtracting them we get

$$
\frac{(\phi-\psi)(\phi \psi(C(\phi+\psi)-D+\beta-\gamma)+\delta(\phi+\psi))}{\phi \psi}=0 .
$$

If we set

$$
\phi+\psi=x \quad \text { and } \phi \psi=y,
$$

where $x, y>0$, then $\phi$ and $\psi$ are positive and different solutions of the quadratic equation

$$
t^{2}-x t+y=0 .
$$

In addition to the conditions $x, y>0$ it is necessary that $x^{2}-4 y>0$. 
From (22) and (23) we get the system

$$
\left\{\begin{array}{l}
y(C x-D+\beta-\gamma)+x \delta=0 \\
y(B-C)+C x^{2}-x \gamma+\delta=0
\end{array}\right.
$$

Theorem 6 For (1) the following holds:

(i) If $\gamma=0$ then (1) has no a minimal period-two solution.

(ii) If $C=0, \gamma>0$ and $\delta>0$ then (1) has the minimal period-two solution $\{\phi, \psi\}$ where

$$
\begin{aligned}
& \phi=\frac{\delta(D-\beta+\gamma+\sqrt{4 B \delta+(D-\beta-3 \gamma)(D-\beta+\gamma)})}{2 \gamma(D-\beta+\gamma)-2 B \delta}, \\
& \psi=\frac{\delta(D-\beta+\gamma-\sqrt{4 B \delta+(D-\beta-3 \gamma)(D-\beta+\gamma)})}{2 \gamma(D-\beta+\gamma)-2 B \delta}
\end{aligned}
$$

if and only if

$$
4 B \delta+(D-\beta-3 \gamma)(D-\beta+\gamma)>0 \text { and } \gamma(D-\beta+\gamma)-B \delta>0
$$

(iii) If $\delta=0, \gamma>0$ and $C>0$ then (1) has two minimal period-two solutions $\{0, \gamma / C\}$ and $\{\phi, \psi\}$ where

$$
\begin{aligned}
& \phi=\frac{(B-C)(D-\beta+\gamma)+\sqrt{D-\beta+\gamma} \sqrt{(B-C)((B+3 C)(D-\beta)+\gamma(B-C))}}{2 C(B-C)}, \\
& \psi=\frac{(B-C)(D-\beta+\gamma)-\sqrt{D-\beta+\gamma} \sqrt{(B-C)((B+3 C)(D-\beta)+\gamma(B-C))}}{2 C(B-C)},
\end{aligned}
$$

if and only if

$$
((B+3 C)(D-\beta)+\gamma(B-C))(B-C)>0 \text { and } \quad D-\beta+\gamma>0 \wedge(B-C)(D-\beta)<0 .
$$

(iv) If $\delta=C=0, \gamma>0$ then (1) has no minimal period-two solution.

Proof (i) The proof follows from (22), since $\phi, \psi \geq 0$ and $\phi \neq \psi$.

(ii) Assume that $C=0$ and $\delta>0$. By using (24) we see that $x$ and $y$ satisfy the following equations:

$$
y(-D+\beta-\gamma)+x \delta=0 \wedge B y=(x \gamma-\delta) .
$$

Assume that $\gamma(D-\beta+\gamma)-B \delta \neq 0$. The solution of system (24) is given by

$$
x=\frac{\delta(D-\beta+\gamma)}{\gamma(D-\beta+\gamma)-B \delta}, \quad y=\frac{\delta^{2}}{\gamma(D-\beta+\gamma)-B \delta},
$$

and $x, y>0 \wedge x^{2}-4 y>0$ if and only if

$$
4 B \delta+(D-\beta-3 \gamma)(D-\beta+\gamma)>0 \wedge \gamma(D-\beta+\gamma)-B \delta>0
$$


since

$$
\begin{aligned}
x^{2}-4 y & =\frac{\delta^{2}(D-\beta+\gamma)^{2}}{(\gamma(D-\beta+\gamma)-B \delta)^{2}}-\frac{4 \delta^{2}}{\gamma(D-\beta+\gamma)-B \delta} \\
& =\frac{\delta^{2}(4 B \delta+(D-\beta-3 \gamma)(D-\beta+\gamma))}{(\gamma(D-\beta+\gamma)-B \delta)^{2}} .
\end{aligned}
$$

In this case the equation

$$
t^{2}-\frac{\delta(D-\beta+\gamma)}{\gamma(D-\beta+\gamma)-B \delta} t+\frac{\delta^{2}}{\gamma(D-\beta+\gamma)-B \delta}=0
$$

has positive distinct solutions which are given by

$$
t_{ \pm}=\frac{\delta(D-\beta+\gamma \pm \sqrt{4 B \delta+(D-\beta-3 \gamma)(D-\beta+\gamma)})}{2 \gamma(D-\beta+\gamma)-2 B \delta} .
$$

If $\gamma(D-\beta+\gamma)-B \delta=0$, it is easy to see that system (27) has no solutions from which follows that (1) has no minimal period-two solution.

(iii) Assume that $\delta=0, \gamma>0$ and $C>0$. By using (24) we find that $x$ and $y$ satisfy the following equations:

$$
\left\{\begin{array}{l}
y(C x-D+\beta-\gamma)=0, \\
y(B-C)+C x^{2}-x \gamma=0 .
\end{array}\right.
$$

Assume that $y \neq 0$ and $B \neq C$. The solution of system (28) is given by

$$
x=\frac{D-\beta+\gamma}{C} \text { and } y=-\frac{(D-\beta)(D-\beta+\gamma)}{C(B-C)},
$$

and $x, y>0 \wedge x^{2}-4 y>0$ if and only if

$$
((B+3 C)(D-\beta)+\gamma(B-C))(B-C)>0 \wedge D-\beta+\gamma>0 \wedge(B-C)(D-\beta)<0,
$$

since

$$
\begin{aligned}
x^{2}-4 y & =\frac{(D-\beta+\gamma)^{2}}{C^{2}}+\frac{4(D-\beta)(D-\beta+\gamma)}{C(B-C)} \\
& =\frac{(D-\beta+\gamma)((B+3 C)(D-\beta)+\gamma(B-C))}{C^{2}(B-C)} .
\end{aligned}
$$

In this case the equation

$$
t^{2}-\frac{D-\beta+\gamma}{C} t-\frac{(D-\beta)(D-\beta+\gamma)}{C(B-C)}=0
$$

has positive distinct solutions which are given by

$$
t_{ \pm}=\frac{(B-C)(D-\beta+\gamma) \pm \sqrt{D-\beta+\gamma} \sqrt{(B-C)((B+3 C)(D-\beta)+\gamma(B-C))}}{2 C(B-C)} .
$$


If $y=0$, then from (28) we have $x=\frac{\gamma}{C}$, which implies that $\left\{\frac{\gamma}{C}, 0\right\}$ is the minimal periodtwo solution.

If $y \neq 0$ and $B=C$, then the rest of the proof follows from Lemma 2 .

(iv) The proof follows from the proof of Lemma 2.

Theorem 7 Assume that $C=B>0, \delta>0$ and $\gamma>0$. Let

$$
x_{ \pm}=\frac{\gamma \pm \sqrt{\gamma^{2}-4 C \delta}}{2 C}, \quad y_{ \pm}=\frac{\delta\left(\left(\gamma \pm \sqrt{\gamma^{2}-4 C \delta}\right)(D-\beta+\gamma)-2 C \delta\right)}{2 C(C \delta+(D-\beta)(D-\beta+\gamma))}
$$

Then for (1) the following holds:

(i) If

$$
\gamma^{2}>4 c \delta \text { and } D>\frac{3}{2}\left(\sqrt{\gamma^{2}-4 C \delta}+\gamma\right)+\beta
$$

then (1) has two minimal period-two solutions $\left\{\phi_{+}, \psi_{+}\right\}$and $\left\{\phi_{-}, \psi_{-}\right\}$, where $\phi_{+}$and $\psi_{+}$are solutions of equation $t^{2}-x_{+} t+y_{+}=0$ and $\phi_{-}$and $\psi_{-}$are solutions of equation $t^{2}-x_{-} t+y_{-}=0$.

(ii) If

$$
\gamma^{2}>4 c \delta \text { and } \frac{3}{2}\left(\gamma-\sqrt{\gamma^{2}-4 C \delta}\right)+\beta<D \leq \frac{3}{2}\left(\gamma+\sqrt{\gamma^{2}-4 C \delta}\right)+\beta
$$

then (1) has one minimal period-two solution $\left\{\phi_{+}, \psi_{+}\right\}$where $\phi_{+}$and $\psi_{+}$are solutions of equation $t^{2}-x_{+} t+y_{+}=0$.

(iii) In all other cases (1) has no minimal period-two solution.

Proof It is clear that $\left(x_{ \pm}, y_{ \pm}\right)$are solutions of system (24). Then minimal period-two solutions are solutions of the equation $t^{2}-x_{+} t+y_{+}=0$ if $x_{+}, y_{+}>0 \wedge x_{+}^{2}-4 y_{+}>0$ and the equation $t^{2}-x_{-} t+y_{-}=0$ if $x_{-}, y_{-}>0 \wedge x_{-}^{2}-4 y_{-}>0$. Let

$$
\begin{aligned}
\Delta= & D^{2}\left(\gamma^{2}-2 C \delta\right)+D\left(4 C \beta \delta-6 C \gamma \delta-2 \beta \gamma^{2}+\gamma^{3}\right) \\
& +\beta^{2}\left(\gamma^{2}-2 C \delta\right)-\beta\left(\gamma^{3}-6 C \gamma \delta\right)+3 C \delta\left(2 C \delta-\gamma^{2}\right)
\end{aligned}
$$

and

$$
\Gamma=D\left(-4 C \delta-2 \beta \gamma+\gamma^{2}\right)+4 C \beta \delta-3 C \gamma \delta+D^{2} \gamma+\beta^{2} \gamma-\beta \gamma^{2}
$$

One can show that the following identities hold:

$$
\begin{aligned}
& x_{ \pm}-4 y_{ \pm}=\frac{\Delta \pm \Gamma \sqrt{\gamma^{2}-4 c \delta}}{C \delta+(D-\beta)(D-\beta+\gamma)}, \\
& \Delta^{2}-\Gamma^{2}\left(\gamma^{2}-4 c \delta\right)=4 C^{2} \delta^{2}(C \delta+(D-\beta)(D-\beta+\gamma))(9 C \delta+(D-\beta)(D-\beta-3 \gamma)),
\end{aligned}
$$

from which the proof follows. 
Assume now that $B \neq C$ and $C>0$. Solving the second equation of system (24) for $y$ we get

$$
y=\frac{-C x^{2}+x \gamma-\delta}{B-C}
$$

Substituting (29) in the first equation of system (24) we see that $x$ satisfies the following equation:

$$
-C^{2} x^{3}+C x^{2}(D-\beta+2 \gamma)+x\left(B \delta-2 C \delta-D \gamma+\beta \gamma-\gamma^{2}\right)+\delta(D-\beta+\gamma)=0 .
$$

In a similar way one can show that $y$ satisfies the following equation:

$$
\begin{aligned}
& C^{2} y^{3}(C-B)+C y^{2}\left(-2 B \delta+C \delta-D^{2}+2 D \beta-D \gamma-\beta^{2}+\beta \gamma\right) \\
& \quad+y \delta\left(-B \delta-C \delta+D \gamma-\beta \gamma+\gamma^{2}\right)-\delta^{3}=0 .
\end{aligned}
$$

Let

$$
\begin{aligned}
& a=-C^{2}, \quad b=C(D-\beta+2 \gamma), \\
& c=\left(B \delta-2 C \delta-D \gamma+\beta \gamma-\gamma^{2}\right), \quad d=-\delta^{3} .
\end{aligned}
$$

The solutions of (30) are given by

$$
\begin{aligned}
& x_{1}=S+T-\frac{b}{3 a}, \quad x_{2}=-\frac{S+T}{2}-\frac{b}{3 a}+\frac{i \sqrt{3}}{2}(S-T), \\
& x_{3}=-\frac{S+T}{2}-\frac{b}{3 a}-\frac{i \sqrt{3}}{2}(S-T),
\end{aligned}
$$

where

$$
S=\sqrt[3]{R+\sqrt{Q^{3}+R^{2}}} \text { and } T=\sqrt[3]{R-\sqrt{Q^{3}+R^{2}}}
$$

and

$$
Q=\frac{3 a c-b^{2}}{9 a^{2}}, \quad R=\frac{9 a b c-27 a^{2} d-2 b^{3}}{54 a^{3}} .
$$

Then the solutions of the system (24) are given by

$$
\left(x_{i}, y_{i}\right)=\frac{-C x_{i}^{2}+x_{i} \gamma-\delta}{B-C}, \quad i=1,2,3
$$

If $x_{i}, y_{i}>0$ and $x_{i}^{2}-4 y_{i}>0$, then (1) has minimal period-two solutions given by

$$
\left\{\phi_{i}=\frac{x_{i}-\sqrt{x_{i}^{2}-4 y_{i}}}{2}, \psi_{i}=\frac{x_{i}+\sqrt{x_{i}^{2}-4 y_{i}}}{2}\right\} \quad \text { for } i=1,2,3 .
$$


Let $g$ be a function given by

$$
\begin{aligned}
g(x)= & C^{2} x^{6}(C-B)-C x^{5}(C-B)(D-\beta+2 \gamma)+x^{4}\left(\delta\left(B^{2}-B C+C^{2}\right)\right. \\
& +\gamma(C-B)(D-\beta+\gamma)+C(D-\beta)(D-\beta+\gamma)) \\
& +x^{3}(\delta(B(2 D-2 \beta+\gamma)-2 C(D-\beta+\gamma))+\gamma(\beta-D)(D-\beta+\gamma)) \\
& +x^{2} \delta\left(-2 B \delta+C \delta+(D-\beta+\gamma)^{2}\right)-x \delta^{2}(2 D-2 \beta+\gamma)+\delta^{3} .
\end{aligned}
$$

Eliminating $\phi$ and $\psi$ from (20) and (21) implies that if $\{\phi, \psi\}$ is a minimal period-two solution; then

$$
g(\phi)=0 \quad \text { and } \quad g(\psi)=0 \quad \text { with } \phi \neq \psi,
$$

from which it follows that

$$
g(t)=C^{2}(C-B)\left(t^{2}-x_{1} t+y_{1}\right)\left(t^{2}-x_{2} t+y_{2}\right)\left(t^{2}-x_{3} t+y_{3}\right)
$$

for $B \neq C$, since $\left\{\phi_{i}, \psi_{i}\right\}$ are distinct roots of equation $t^{2}-x_{i} t+y_{i}=0$.

\section{Local stability of period-two solutions}

Let $\{\phi, \psi\}$ be a minimal period-two solution of (1).

Set

$$
u_{n}=x_{n-1} \quad \text { and } \quad v_{n}=x_{n} \quad \text { for } n=0,1, \ldots
$$

and write (1) in the equivalent form

$$
\begin{aligned}
& u_{n+1}=v_{n}, \\
& v_{n+1}=\frac{\beta v_{n} u_{n}+\gamma u_{n}^{2}+\delta v_{n}}{B u_{n} v_{n}+C u_{n}^{2}+D v_{n}}
\end{aligned}
$$

for $n=0,1, \ldots$. Let $T$ be the function defined by

$$
T(u, v)=\left(v, \frac{\beta v u+\gamma u^{2}+\delta v}{B u v+C u^{2}+D v}\right) .
$$

Then $\{\phi, \psi\}$ is a fixed point of $T^{2}$, the second iterate of $T$, and

$$
T^{2}(u, v)=(G(u, v), H(u, v))
$$

where $H(u, v)=G(v, G(u, v))$ and

$$
\begin{aligned}
G(u, v) & =\frac{u^{2} \gamma+u v \beta+v \delta}{B u v+C u^{2}+D v} \\
H(u, v) & =\frac{u v\left(v\left(B v \gamma+\beta^{2}\right)+\beta \delta\right)+u^{2} \gamma(v(C v+\beta)+\delta)+v\left(D v^{2} \gamma+\delta(v \beta+\delta)\right)}{C v^{3}(B u+D)+(B v+D)\left(u^{2} \gamma+u v \beta+v \delta\right)+C^{2} u^{2} v^{2}} .
\end{aligned}
$$


By definition

$$
J_{T^{2}}(\phi, \psi)=\left(\begin{array}{ll}
\frac{\partial G}{\partial u}(\phi, \psi) & \frac{\partial G}{\partial v}(\phi, \psi) \\
\frac{\partial H}{\partial u}(\phi, \psi) & \frac{\partial H}{\partial v}(\phi, \psi)
\end{array}\right) .
$$

Theorem 8 If $C=0, \gamma>0$ and $\delta>0$, then (1) has the minimal period-two solution $\{\phi, \psi\}$ where $\phi$ and $\psi$ are given by (25) and (26) if and only if

$$
4 B \delta+(D-\beta-3 \gamma)(D-\beta+\gamma)>0 \wedge \gamma(D-\beta+\gamma)-B \delta>0 .
$$

In this case the minimal period-two solution $\{\phi, \psi\}$ is a saddle point.

Proof The existence of the minimal period-two solution follows from Theorem 6. Now, we prove that the minimal period-two solution is a saddle point. Since $G(\phi, \psi)=\phi$ we have

$$
\delta=\frac{\phi(\psi(B \phi+D-\beta)-\gamma \phi)}{\psi} .
$$

Using (31) and the fact $C=0$ we see that the Jacobian matrix of $T^{2}$ at the point $\{\phi, \psi\}$ is given by

$$
\begin{aligned}
& J_{T^{2}}(\phi, \psi) \\
& \quad=\left(\begin{array}{cc}
\frac{2 \gamma \phi-B \psi \phi+\beta \psi}{(D+B \phi) \psi} & -\frac{\gamma \phi^{2}}{(D+B \phi) \psi^{2}} \\
\frac{\gamma \psi(-2 \gamma \phi+B \psi(\phi-\beta \psi)}{\phi^{2}(D+B \phi)(D+B \psi)} & \frac{\phi \psi(D+B \psi) \gamma^{2}+(D+B \phi)\left(2 D \psi^{2}+B\left(\phi^{3} \psi^{3}\right)\right) \gamma-\phi(D+B \phi)^{2}(B \phi-\beta) \psi}{\phi(D+B \phi) \psi(D+B \psi)^{2}}
\end{array}\right) .
\end{aligned}
$$

The determinant of the Jacobian matrix (32) is given by

$$
\begin{aligned}
& \operatorname{det} J_{T^{2}}(\phi, \psi) \\
& \quad=\frac{(B \phi \psi-\beta \psi-2 \gamma \phi)\left(\phi \psi(B \phi+D)(B \phi-\beta)-\gamma\left(B\left(\phi^{3}+\psi^{3}\right)+2 D \psi^{2}\right)\right)}{\phi \psi^{2}(B \phi+D)(B \psi+D)^{2}} .
\end{aligned}
$$

The trace of the Jacobian matrix (32) is given by

$$
\begin{aligned}
& \operatorname{tr} J_{T^{2}}(\phi, \psi) \\
& \quad=\frac{\frac{-\phi \psi(B \phi+D)^{2}(B \phi-\beta)+\gamma^{2} \phi \psi(B \psi+D)+\gamma(B \phi+D)\left(B\left(\phi^{3}+\psi^{3}\right)+2 D \psi^{2}\right)}{\phi(B \psi+D)^{2}}-B \phi \psi+\beta \psi+2 \gamma \phi}{\psi(B \phi+D)} .
\end{aligned}
$$

Substituting (25) and (26) into (33) and (34) we find that the determinant of the Jacobian matrix (32) is given by

$$
\begin{aligned}
& \operatorname{det} J_{T^{2}}(\phi, \psi) \\
& \quad=\frac{B^{2} \delta^{2}-B \delta(D(\beta+2 \gamma)+\gamma(6 \gamma-5 \beta))+\gamma(D-\beta+\gamma)\left(2 D \beta-\beta^{2}-2 \beta \gamma+4 \gamma^{2}\right)}{B^{2} \delta^{2}+B D \delta(\gamma-\beta)+D^{2} \gamma(D-\beta+\gamma)},
\end{aligned}
$$


and the trace of the Jacobian matrix (32) is given by

$$
\begin{aligned}
& \operatorname{tr} J_{T^{2}}(\phi, \psi) \\
& \quad=\frac{-2 B^{2} \delta^{2}-B \delta\left(D^{2}-5 D \gamma+\beta^{2}+\gamma(\beta-\gamma)\right)+\gamma\left(2 D^{2}-2 D \gamma+\gamma^{2}\right)(D-\beta+\gamma)}{B^{2} \delta^{2}+B D \delta(\gamma-\beta)+D^{2} \gamma(D-\beta+\gamma)} .
\end{aligned}
$$

The period-two solution $\{\phi, \psi\}$ is a saddle point if and only if

$$
\left|\operatorname{tr} J_{T^{2}}(\phi, \psi)\right|>\left|1+\operatorname{det} J_{T^{2}}(\phi, \psi)\right| .
$$

One can see that

$$
1+\operatorname{det} J_{T^{2}}(\phi, \psi)-\operatorname{tr} J_{T^{2}}(\phi, \psi)=-\frac{(\gamma(D-\beta+\gamma)-B \delta)(4 B \delta+(D-\beta-3 \gamma)(D-\beta+\gamma))}{B^{2} \delta^{2}+B D \delta(\gamma-\beta)+D^{2} \gamma(D-\beta+\gamma)}
$$

and

$$
\begin{aligned}
1+\operatorname{det} J_{T^{2}}(\phi, \psi)+\operatorname{tr} J_{T^{2}}(\phi, \psi)= & \frac{\gamma(D-\beta+\gamma)\left(3 D^{2}-2 \gamma(D+\beta)+2 D \beta-\beta^{2}+5 \gamma^{2}\right)}{B^{2} \delta^{2}+B D \delta(\gamma-\beta)+D^{2} \gamma(D-\beta+\gamma)} \\
& -\frac{B \delta\left(-4 \gamma(D+\beta)+(D+\beta)^{2}+5 \gamma^{2}\right)}{B^{2} \delta^{2}+B D \delta(\gamma-\beta)+D^{2} \gamma(D-\beta+\gamma)} .
\end{aligned}
$$

Since

$$
\gamma(D-\beta+\gamma)-B \delta>0
$$

we have

$$
\beta<\frac{-B \delta+D \gamma+\gamma^{2}}{\gamma} \text {. }
$$

Since

$$
\frac{B^{2} \delta^{2}+B D \gamma \delta+D^{3} \gamma+D^{2} \gamma^{2}}{D(B \delta+D \gamma)}-\frac{-B \delta+D \gamma+\gamma^{2}}{\gamma}=\frac{B^{2} \delta^{2}(D+\gamma)}{D \gamma(B \delta+D \gamma)} \geq 0
$$

we have

$$
\frac{B^{2} \delta^{2}+B D \gamma \delta+D^{3} \gamma+D^{2} \gamma^{2}}{D(B \delta+D \gamma)}>\beta
$$

which implies

$$
B^{2} \delta^{2}+B D \delta(\gamma-\beta)+D^{2} \gamma(D-\beta+\gamma)>0
$$

Hence, we prove that $1+\operatorname{det} J_{T^{2}}(\phi, \psi)-\operatorname{tr} J_{T^{2}}(\phi, \psi)<0$. From (35) we have

$$
B<\frac{\gamma(D-\beta+\gamma)}{\delta} .
$$


Let $h(x)=x^{2}-4 \gamma x+5 \gamma^{2}$. Since the discriminant of $h$ is negative we have $h(x)>0$ for $x \in \mathcal{R}$, which implies

$$
(D+\beta)^{2}-4 \gamma(D+\beta)+5 \gamma^{2}>0 .
$$

Since

$$
\begin{aligned}
& \frac{\gamma(D-\beta+\gamma)\left(3 D^{2}-2 \gamma(D+\beta)+2 D \beta-\beta^{2}+5 \gamma^{2}\right)}{\delta\left(-4 \gamma(D+\beta)+(D+\beta)^{2}+5 \gamma^{2}\right)}-\frac{\gamma(D-\beta+\gamma)}{\delta} \\
& =\frac{2 \gamma(D+\beta)(D-\beta+\gamma)^{2}}{\delta\left(-4 \gamma(D+\beta)+(D+\beta)^{2}+5 \gamma^{2}\right)} \geq 0,
\end{aligned}
$$

we have

$$
\frac{\gamma(D-\beta+\gamma)\left(3 D^{2}-2 \gamma(D+\beta)+2 D \beta-\beta^{2}+5 \gamma^{2}\right)}{\delta\left(-4 \gamma(D+\beta)+(D+\beta)^{2}+5 \gamma^{2}\right)}>B .
$$

Inequality (36) is equivalent to

$$
\begin{gathered}
\gamma(D-\beta+\gamma)\left(3 D^{2}-2 \gamma(D+\beta)+2 D \beta-\beta^{2}+5 \gamma^{2}\right) \\
-B \delta\left(-4 \gamma(D+\beta)+(D+\beta)^{2}+5 \gamma^{2}\right)>0,
\end{gathered}
$$

from which it follows that

$$
1+\operatorname{det} J_{T^{2}}(\phi, \psi)+\operatorname{tr} J_{T^{2}}(\phi, \psi)>0
$$

Theorem 9 Assume $\delta=0, \gamma>0$ and $C>0$. Then (1) has the minimal period-two solution $\{\phi, \psi\}$ where

$$
\begin{aligned}
& \phi=\frac{(B-C)(D-\beta+\gamma)+\sqrt{D-\beta+\gamma} \sqrt{(B-C)((B+3 C)(D-\beta)+\gamma(B-C))}}{2 C(B-C)}, \\
& \psi=\frac{(B-C)(D-\beta+\gamma)-\sqrt{D-\beta+\gamma} \sqrt{(B-C)((B+3 C)(D-\beta)+\gamma(B-C))}}{2 C(B-C)}
\end{aligned}
$$

if and only if

$$
((B+3 C)(D-\beta)+\gamma(B-C))(B-C)>0 \wedge D-\beta+\gamma>0 \wedge(B-C)(D-\beta)<0 .
$$

The minimal period-two solution $\{\phi, \psi\}$ is

(i) locally asymptotically stable if

$$
D-\beta+\gamma>0 \wedge D<\beta \wedge(B+3 C)(D-\beta)+\gamma(B-C)>0,
$$

(ii) a saddle point if

$$
\gamma+3 \beta-3 D>0 \wedge D>\beta \wedge(B+3 C)(D-\beta)+\gamma(B-C)<0 .
$$


Proof The existence of the minimal period-two solution follows from Theorem 6. Now, we investigate the stability of $\{\phi, \psi\}$. The Jacobian matrix of $T^{2}$ at the point $\{\phi, \psi\}$ is given by

$$
J_{T^{2}}(\phi, \psi)=\left(\begin{array}{ll}
e & f \\
g & h
\end{array}\right),
$$

where

$$
\begin{aligned}
e= & \frac{\psi\left(-C \beta \phi^{2}+\gamma(2 D+B \phi) \phi+D \beta \psi\right)}{\left(C \phi^{2}+(D+B \phi) \psi\right)^{2}}, \quad f=\frac{\phi^{2}(C \beta \phi-\gamma(D+B \phi))}{\left(C \phi^{2}+(D+B \phi) \psi\right)^{2}}, \\
g= & \frac{\psi^{3}\left(-C \beta \phi^{2}+\gamma(2 D+B \phi) \phi+D \beta \psi\right)(C \beta \psi-\gamma(D+B \psi))}{\left(C(D+B \phi) \psi^{3}+C^{2} \phi^{2} \psi^{2}+\phi(D+B \psi)(\gamma \phi+\beta \psi)\right)^{2}}, \\
h= & \left(\phi \left(\beta^{2} \psi^{2}\left(D \beta \phi-C \psi^{2}(B \phi+D)\right)+\gamma^{2} \phi\left(C \phi^{2} \psi(B \psi+2 D)\right.\right.\right. \\
& \left.\left.\left.+\psi^{2}(B \phi+D)(2 B \psi+3 D)+D \beta \phi^{2}\right)\right)\right) \\
& /\left(\left(C \psi^{3}(B \phi+D)+\phi(B \psi+D)(\beta \psi+\gamma \phi)+C^{2} \phi^{2} \psi^{2}\right)^{2}\right) \\
& +\frac{\beta \gamma \phi \psi\left(\psi\left(2 \psi(B \phi+D)(D-C \phi)+B \psi^{2}(B \phi+D)+C \phi^{2}(D-C \phi)\right)+2 D \beta \phi^{2}\right)}{\left(C \psi^{3}(B \phi+D)+\phi(B \psi+D)(\beta \psi+\gamma \phi)+C^{2} \phi^{2} \psi^{2}\right)^{2}} .
\end{aligned}
$$

The determinant of the Jacobian matrix (37) is given by

$$
\begin{aligned}
\operatorname{det} J_{T^{2}}(\phi, \psi)= & e h-g f \\
= & \frac{\phi \psi(\beta \psi+\gamma \phi)\left(-\gamma \phi(B \phi+2 D)+C \beta \phi^{2}-D \beta \psi\right)}{\left(\psi(B \phi+D)+C \phi^{2}\right)^{2}} \\
& \times \frac{\beta \psi\left(C \psi\left(\psi(B \phi+D)+C \phi^{2}\right)-D \beta \phi\right)}{\left(C \psi^{3}(B \phi+D)+\phi(B \psi+D)(\beta \psi+\gamma \phi)+C^{2} \phi^{2} \psi^{2}\right)^{2}} \\
& -\frac{\gamma\left(\psi(B \psi+2 D)\left(\psi(B \phi+D)+C \phi^{2}\right)+D \beta \phi^{2}\right)}{\left(C \psi^{3}(B \phi+D)+\phi(B \psi+D)(\beta \psi+\gamma \phi)+C^{2} \phi^{2} \psi^{2}\right)^{2}} .
\end{aligned}
$$

By using

$$
\begin{aligned}
& \phi=\frac{(B-C)(D-\beta+\gamma)+\sqrt{D-\beta+\gamma} \sqrt{(B-C)((B+3 C)(D-\beta)+\gamma(B-C))}}{2 C(B-C)}, \\
& \psi=\frac{(B-C)(D-\beta+\gamma)-\sqrt{D-\beta+\gamma} \sqrt{(B-C)((B+3 C)(D-\beta)+\gamma(B-C))}}{2 C(B-C)}
\end{aligned}
$$

we find that

$$
\begin{aligned}
& \operatorname{det} J_{T^{2}}(\phi, \psi) \\
& =-\frac{(D-\beta)\left(B^{2} D(D-\beta+\gamma)+B C(2 D-\beta)(D-\gamma)-C^{2}(\beta-2 D)^{2}\right)}{(B-C)\left(\gamma^{2}(B \beta-C D)+\beta \gamma(B+C)(D-\beta)+C \beta^{2}(\beta-D)\right)},
\end{aligned}
$$




$$
\begin{aligned}
\operatorname{tr} J_{T^{2}}(\phi, \psi)= & e+h \\
= & \frac{(D-\beta)\left(B^{2} \beta(\beta-D)+B C \beta(\beta-3 D)+C^{2}\left(D^{2}+2 D \beta-\beta^{2}\right)\right)}{(B-C)\left(\gamma^{2}(B \beta-C D)+\beta \gamma(B+C)(D-\beta)+C \beta^{2}(\beta-D)\right)} \\
& +\frac{\gamma(D-\beta)\left(B^{2}(D-\beta)+B C(2 D-\beta)+C^{2}(\beta-2 D)\right)}{(B-C)\left(\gamma^{2}(B \beta-C D)+\beta \gamma(B+C)(D-\beta)+C \beta^{2}(\beta-D)\right)} \\
& +\frac{\gamma^{2}(B-C)(B D+C(\beta-2 D))}{(B-C)\left(\gamma^{2}(B \beta-C D)+\beta \gamma(B+C)(D-\beta)+C \beta^{2}(\beta-D)\right)} .
\end{aligned}
$$

From (38) and (39) it follows that

$$
\begin{aligned}
1+\operatorname{det} J_{T^{2}}(\phi, \psi)-\operatorname{tr} J_{T^{2}}(\phi, \psi)=\frac{(D-\beta)(D-\beta+\gamma)((B+3 C)(D-\beta)+\gamma(B-C))}{\gamma^{2}(C D-B \beta)+\beta \gamma(B+C)(\beta-D)+C \beta^{2}(D-\beta)} & \\
1+\operatorname{det} J_{T^{2}}(\phi, \psi)+\operatorname{tr} J_{T^{2}}(\phi, \psi) & \\
= & \frac{(\beta-D)\left(D^{2}\left(B^{2}+2 B C-5 C^{2}\right)-\beta^{2}\left(B^{2}+C^{2}\right)+2 C D \beta(B+C)\right)}{(B-C)\left(\gamma^{2}(B \beta-C D)+\beta \gamma(B+C)(D-\beta)+C \beta^{2}(\beta-D)\right)} \\
& \times \frac{\gamma^{2}(B-C)(B(D+\beta)+C(\beta-3 D))-2 C \gamma(D-\beta)(B(\beta-2 D)+C D)}{(B-C)\left(\gamma^{2}(B \beta-C D)+\beta \gamma(B+C)(D-\beta)+C \beta^{2}(\beta-D)\right)} \\
1-\operatorname{det} J_{T^{2}}(\phi, \psi) & \frac{\gamma(D-\beta)\left(B^{2}(D+\beta)+B C(\beta-2 D)-C^{2} \beta\right)}{(B-C)\left(\gamma^{2}(B \beta-C D)+\beta \gamma(B+C)(D-\beta)+C \beta^{2}(\beta-D)\right)} \\
& +\frac{(D-\beta)^{2}\left(B^{2} D+B C(2 D+\beta)-4 C^{2} D\right)+\gamma^{2}(B-C)(B \beta-C D)}{(B-C)\left(\gamma^{2}(B \beta-C D)+\beta \gamma(B+C)(D-\beta)+C \beta^{2}(\beta-D)\right)}
\end{aligned}
$$

The rest of the proof follows from Lemma 4.

Lemma 3 Assume that $D-\beta+\gamma>0$.

(1) If $D<\beta$ then $4 D^{2}-5 D \beta+\beta^{2}+5 \beta \gamma+\gamma^{2}>0$.

(2) $2 D^{2}-D(\beta+2 \gamma)-3 \beta^{2}-5 \beta \gamma-\gamma^{2}<0$.

\section{Proof}

(1) It is sufficient to prove that the inequality $4 D^{2}-5 D \beta+\beta^{2}+5 \beta \gamma>0$ holds for $D-\beta+\gamma>0$ and $D<\beta$. Since

$$
\frac{-4 D^{2}+5 D \beta-\beta^{2}}{5 \beta}-(\beta-D)=-\frac{2(2 D-3 \beta)(D-\beta)}{5 \beta}<0
$$

we find that

$$
\frac{-4 D^{2}+5 D \beta-\beta^{2}}{5 \beta}<\beta-D<\gamma
$$

which implies

$$
\frac{-4 D^{2}+5 D \beta-\beta^{2}}{5 \beta}-\gamma=\frac{-4 D^{2}+5 D \beta-\beta^{2}-5 \beta \gamma}{5 \beta}<0,
$$

from which the inequality follows. 
(2) In view of the assumption of the lemma we have

$$
\begin{aligned}
2 D^{2} & -D(\beta+2 \gamma)-3 \beta^{2}-5 \beta \gamma-\gamma^{2} \\
& =2 D(D-\gamma)-D \beta-3 \beta^{2}-5 \beta \gamma-\gamma^{2} \\
& <D \beta-3 \beta^{2}-5 \beta \gamma-\gamma^{2}<(\beta+\gamma) \beta-3 \beta^{2}-5 \beta \gamma-\gamma^{2} \\
& =-2 \beta^{2}-4 \beta \gamma-\gamma^{2}<0,
\end{aligned}
$$

from which the proof follows.

Lemma 4 If $C>0, \gamma>0$ and

$$
((B+3 C)(D-\beta)+\gamma(B-C))(B-C)>0 \wedge D-\beta+\gamma>0 \wedge(B-C)(D-\beta)<0,
$$

then

(i) $(B-C)\left(\gamma^{2}(B \beta-C D)+\beta \gamma(B+C)(D-\beta)+C \beta^{2}(\beta-D)\right)>0$;

(ii) $\gamma(D-\beta)\left(B^{2}(D+\beta)+B C(\beta-2 D)-C^{2} \beta\right)+(D-\beta)^{2}\left(B^{2} D+B C(2 D+\beta)-4 C^{2} D\right)+$ $\gamma^{2}(B-C)(B \beta-C D)>0$

(iii) $(\beta-D)\left(D^{2}\left(B^{2}+2 B C-5 C^{2}\right)-\beta^{2}\left(B^{2}+C^{2}\right)+2 C D \beta(B+C)\right)+\gamma^{2}(B-C)(B(D+\beta)+$ $C(\beta-3 D))-2 C \gamma(D-\beta)(B(\beta-2 D)+C D)>0$.

Proof (i) Assume that $D>\beta$ and $\beta>0$ holds. Observe that

$$
\frac{-C D \beta^{2}+C D \beta \gamma-C D \gamma^{2}+C \beta^{3}-C \beta^{2} \gamma}{\beta \gamma(-D+\beta-\gamma)}-C=\frac{C(D-\beta)(\beta-\gamma)^{2}}{\beta \gamma(D-\beta+\gamma)} \geq 0 .
$$

Since $B<C$, we obtain

$$
\begin{aligned}
& \frac{-C D \beta^{2}+C D \beta \gamma-C D \gamma^{2}+C \beta^{3}-C \beta^{2} \gamma}{\beta \gamma(-D+\beta-\gamma)}-B \\
& =\frac{\gamma^{2}(B \beta-C D)+\beta \gamma(B+C)(D-\beta)+C \beta^{2}(\beta-D)}{\beta \gamma(-D+\beta-\gamma)} \geq 0 .
\end{aligned}
$$

Since $D-\beta+\gamma>0$, we obtain

$$
\gamma^{2}(B \beta-C D)+\beta \gamma(B+C)(D-\beta)+C \beta^{2}(\beta-D)<0,
$$

which implies

$$
(B-C)\left(\gamma^{2}(B \beta-C D)+\beta \gamma(B+C)(D-\beta)+C \beta^{2}(\beta-D)\right)>0 .
$$

Similarly, one can prove the statement of the lemma if $(D<\beta$ and $\beta>0)$ or $\beta=0$.

(ii) Assume $D>\beta$. The lemma's assumptions imply

$$
B<C \text { and } B<\frac{C(-3 D+3 \beta+\gamma)}{D-\beta+\gamma} \text { and }-3 D+3 \beta+\gamma>0 \text {. }
$$


Assume for the sake of contradiction that $D-\beta-\gamma \geq 0$. Then $-3 D+3 \beta+\gamma>0$ and $D-\beta-\gamma \geq 0$ imply $D<\beta$, which is a contradiction. Hence, $D-\beta-\gamma<0$. Let

$$
\begin{aligned}
\Delta_{1}= & (D-\beta)^{2}\left(20 D^{4}+D^{3}(8 \gamma-36 \beta)+D^{2}\left(13 \beta^{2}+12 \beta \gamma-4 \gamma^{2}\right)\right. \\
& \left.+2 D \beta\left(\beta^{2}-9 \beta \gamma+5 \gamma^{2}\right)+\left(\beta^{2}-\beta \gamma+\gamma^{2}\right)^{2}\right)
\end{aligned}
$$

and

$$
\begin{aligned}
H_{1}(B):= & \gamma(D-\beta)\left(B^{2}(D+\beta)+B C(\beta-2 D)-C^{2} \beta\right) \\
& +(D-\beta)^{2}\left(B^{2} D+B C(2 D+\beta)-4 C^{2} D\right)+\gamma^{2}(B-C)(B \beta-C D) .
\end{aligned}
$$

The solutions of the equation $H_{1}(B)=0$ for $B$ are given by

$$
B_{ \pm}=\frac{C\left(-2 D^{3}+3 D^{2} \beta+2 D^{2} \gamma-3 D \beta \gamma+D \gamma^{2}-\beta^{3}+\beta^{2} \gamma+\beta \gamma^{2} \pm \sqrt{\Delta_{1}}\right)}{2(D-\beta+\gamma)\left(D^{2}-D \beta+\beta \gamma\right)} .
$$

Since the coefficient of $B^{2}$ in the quadratic equation $H_{1}(B)=0$ is positive we have $H_{1}(B)>$ $0 \Leftrightarrow B \in\left[0, B_{-}\right) \cup\left(B_{+}, \infty\right)$.

Note that the following three identities hold:

$$
\begin{aligned}
& 4 D^{2}-5 D \beta+\beta^{2}+5 \beta \gamma+\gamma^{2}=(\beta+\gamma-D)(\beta+\gamma+D)+5 D(D-\beta)+3 \beta \gamma ; \\
& \Delta_{1}-(D-\beta)^{2}\left(4 D^{2}-5 D \beta+\beta^{2}+5 \beta \gamma+\gamma^{2}\right)^{2} \\
& \quad=4(D-\beta)^{2}(D-\beta-\gamma)\left(D^{2}-D \beta+\beta \gamma\right)(D+3(\beta+\gamma)) ; \\
& B_{ \pm}-\frac{C(-3 D+3 \beta+\gamma)}{D-\beta+\gamma}=\frac{C\left((D-\beta)\left(4 D^{2}-5 D \beta+\beta^{2}+5 \beta \gamma+\gamma^{2}\right) \pm \sqrt{\Delta_{1}}\right)}{2(D-\beta+\gamma)\left(D^{2}-D \beta+\beta \gamma\right)} .
\end{aligned}
$$

If $\Delta_{1}<0$ then $H_{1}(B)>0$ for all $B \geq 0$. Assume $\Delta_{1} \geq 0$ holds. Since

$$
B<\frac{C(-3 D+3 \beta+\gamma)}{D-\beta+\gamma} \text { and } D>\beta \text { and } D-\beta-\gamma<0
$$

from (41), (42) and (43) we have $B_{-}>\frac{C(-3 D+3 \beta+\gamma)}{D-\beta+\gamma}>B$, which implies that $H_{1}(B)>0$.

Similarly, if $D<\beta$ the assumption of the lemma implies

$$
B>C \text { and } B>\frac{C(-3 D+3 \beta+\gamma)}{D-\beta+\gamma} \text { and } D-\beta+\gamma>0 \text {, }
$$

from which it follows that

$$
D^{2}-D \beta+\beta \gamma>D^{2}-D \beta+D \gamma=D(D-\beta+\gamma) \geq 0 \text {. }
$$

In view of Lemma 3, (43), and (44) we get

$$
B_{+}<\frac{C(-3 D+3 \beta+\gamma)}{D-\beta+\gamma}<B
$$

which implies $H_{1}(B)>0$ for $\frac{C(-3 D+3 \beta+\gamma)}{D-\beta+\gamma}<B$, from which the proof follows. 
(iii) Assume that $D>\beta$. As in case (ii) we have

$$
B<\frac{C(-3 D+3 \beta+\gamma)}{D-\beta+\gamma} \text { and } D-\beta-\gamma<0 .
$$

Let

$$
\begin{aligned}
\Delta_{2}= & (D-\beta)^{2}\left(6 D^{4}+\gamma^{2}\left(6 D^{2}-2 D \beta+\beta^{2}\right)\right. \\
& \left.-3 D^{2} \beta^{2}+2 D \beta^{3}-2 D \gamma(3 D-2 \beta)(D+\beta)-6 D \gamma^{3}-\beta^{4}+\gamma^{4}\right)
\end{aligned}
$$

and

$$
\begin{aligned}
H_{2}(B):= & (\beta-D)\left(D^{2}\left(B^{2}+2 B C-5 C^{2}\right)-\beta^{2}\left(B^{2}+C^{2}\right)+2 C D \beta(B+C)\right) \\
& +\gamma^{2}(B-C)(B(D+\beta)+C(\beta-3 D))-2 C \gamma(D-\beta)(B(\beta-2 D)+C D) .
\end{aligned}
$$

The solutions of the equation $H_{2}(B)=0$ for $B$ are given by

$$
B_{ \pm}=\frac{C\left(-D^{3}+2 D^{2} \gamma+D\left(\beta^{2}-3 \beta \gamma-2 \gamma^{2}\right)+\beta^{2} \gamma \pm \sqrt{\Delta_{2}}\right)}{(D+\beta)(D-\beta-\gamma)(D-\beta+\gamma)} .
$$

Note that the following two identities hold:

$$
\begin{aligned}
\Delta_{2}- & (D-\beta)^{2}\left(2 D^{2}-D(\beta+2 \gamma)-3 \beta^{2}-5 \beta \gamma-\gamma^{2}\right)^{2} \\
& =2(D-\beta)^{2}(D+\beta)(D-\beta-\gamma)\left(D^{2}+2 D \beta+2 D \gamma+5 \beta^{2}+10 \beta \gamma+5 \gamma^{2}\right), \\
B_{ \pm}- & \frac{C(-3 D+3 \beta+\gamma)}{D-\beta+\gamma} \\
& =\frac{C\left((D-\beta)\left(2 D^{2}-D(\beta+2 \gamma)-3 \beta^{2}-5 \beta \gamma-\gamma^{2}\right) \pm \sqrt{\Delta_{2}}\right)}{(D+\beta)(D-\beta-\gamma)(D-\beta+\gamma)} .
\end{aligned}
$$

In view of (45), (46), (47), $D>\beta, D-\beta-\gamma<0$, which by Lemma 3 implies that $B_{-}>$ $B_{+}>B$. This and the fact that the coefficient of $B^{2}$ is positive imply $H_{2}(B)>0$.

Assume that $D<\beta$ and for the sake of contradiction that $D-\beta-\gamma \geq 0$. Then, adding the inequalities $D-\beta+\gamma>0$ and $D-\beta-\gamma \geq 0$, we obtain $D>\beta$, which is a contradiction. Hence, $D-\beta-\gamma<0$. Similarly, by using (45), (46), (47), and Lemma 3, one can prove statement (iii) if $D<\beta$, and the proof will be omitted.

\section{Boundedness of solutions of (1)}

In view of Theorems 1 and 2 and Corollaries 1 and 2, any result on the existence of lower and upper bounds of the solutions of (1) yields some global asymptotic stability result for the unique equilibrium of (1). See Section 6 for such results. As we show in Remark 1 global asymptotic results obtained by application of Theorems 1 and 2 and Corollaries 1 and 2 are not sharp, in the sense that they do not cover the whole parametric region of global asymptotic stability, but they are robust as they can be applied as soon as we have the lower and upper bounds of the solutions. The problem of boundedness of all solutions of (1) is more difficult than the corresponding problem for the linear fractional equation and in view of its importance requires a separate paper. An additional difficulty in studying boundedness is the presence of quadratic terms. 
Here we will give some equations for which the boundedness of all solutions is clear and leave the problem of determining the boundedness of all solutions for a future study. The boundedness or existence of unbounded solutions for all nine special cases of (1) of the type $(1,1)$ follows immediately from the corresponding properties of the linear equation obtained by the substitution $x_{n}=e^{u_{n}}$ and it can range from boundedness of all solutions, periodicity of all solutions with the same period, to the unboundedness of all solutions. All three special cases of $(1)$ of the type $(1,3)$, and all three special cases of $(1)$ of the type $(2,3)$ as well as the special case of the type $(3,3)$ have all solutions uniformly bounded that is, there are constants $L, U, L<U$ such that every solution satisfies $L \leq x_{n} \leq U, n=1,2, \ldots$. All six special cases of $(1)$ of the type $(1,2)$, where the term in the nominator is also present in the denominator are also uniformly bounded as well as three special cases of (1) of the type $(2,2)$, with corresponding terms in the nominator and the denominator. Two special cases of (1), where $B=C=0$ and $B=C=\gamma=0$ allow the existence of unbounded solutions. The remaining 22 special cases of (1) require detailed study and probably new methods in determining boundedness of solutions and complete classification of all special cases of (1).

\section{Global asymptotic stability results}

In this section we give the following global asymptotic stability result for some special cases of (1).

\section{Theorem 10}

(i) Consider (1), where $D=0$, and all other coefficients are positive, subject to the condition

$$
\frac{(|\beta-B \bar{x}|+|\gamma-C \bar{x}|)(U+\bar{x})+\delta}{(B+C) L^{2}}<1,
$$

where $L=\frac{\min \{\beta, \gamma, \delta\}}{\max \{B, C\}}, U=\frac{\max \{\beta, \gamma\}}{\min \{B, C\}}+\frac{\delta}{B L}$. Then $\bar{x}$ is globally asymptotically stable.

(ii) Consider (1), where all coefficients are positive, subject to condition (5), where $L=\frac{\min \{\beta, \gamma, \delta\}}{\max \{B, C, D\}}, U=\frac{\max \{\beta, \gamma, \delta\}}{\min \{B, C, D\}}$. Then $\bar{x}$ is globally asymptotically stable.

Proof In view of Corollary 1 we need to find the lower and upper bounds for all solutions of (1) for $n \geq 1$.

(i) In this case the lower and upper bounds for all solutions of (1) for $n \geq 1$ are derived as

$$
x_{n+1}=\frac{\beta x_{n} x_{n-1}+\gamma x_{n-1}^{2}+\delta x_{n}}{B x_{n} x_{n-1}+C x_{n-1}^{2}} \geq \frac{\min \{\beta, \gamma, \delta\}}{\max \{B, C\}} \frac{x_{n-1}^{2}+x_{n} x_{n-1}+x_{n}}{x_{n-1}^{2}+x_{n} x_{n-1}} \geq \frac{\min \{\beta, \gamma, \delta\}}{\max \{B, C\}}=L>0,
$$

and

$$
\begin{aligned}
x_{n+1} & =\frac{\beta x_{n} x_{n-1}+\gamma x_{n-1}^{2}+\delta x_{n}}{B x_{n} x_{n-1}+C x_{n-1}^{2}}=\frac{\beta x_{n} x_{n-1}+\gamma x_{n-1}^{2}}{B x_{n} x_{n-1}+C x_{n-1}^{2}}+\frac{\delta x_{n}}{B x_{n} x_{n-1}+C x_{n-1}^{2}} \\
& \leq \frac{\max \{\beta, \gamma\}}{\min \{B, C\}} \frac{x_{n-1}^{2}+x_{n} x_{n-1}}{x_{n-1}^{2}+x_{n} x_{n-1}}+\frac{\delta}{B x_{n-1}} \leq \frac{\max \{\beta, \gamma\}}{\min \{B, C\}}+\frac{\delta}{B L}=U .
\end{aligned}
$$


(ii) In this case the lower and upper bounds for all solutions of (1) for $n \geq 1$ are derived as

$$
\begin{aligned}
x_{n+1} & =\frac{\beta x_{n} x_{n-1}+\gamma x_{n-1}^{2}+\delta x_{n}}{B x_{n} x_{n-1}+C x_{n-1}^{2}+D x_{n}} \geq \frac{\min \{\beta, \gamma, \delta\}}{\max \{B, C, D\}} \frac{x_{n-1}^{2}+x_{n} x_{n-1}+x_{n}}{x_{n-1}^{2}+x_{n} x_{n-1}+x_{n}} \\
& =\frac{\min \{\beta, \gamma, \delta\}}{\max \{B, C, D\}}=L>0,
\end{aligned}
$$

and

$$
\begin{aligned}
x_{n+1} & =\frac{\beta x_{n} x_{n-1}+\gamma x_{n-1}^{2}+\delta x_{n}}{B x_{n} x_{n-1}+C x_{n-1}^{2}+D x_{n}} \leq \frac{\max \{\beta, \gamma, \delta\}}{\min \{B, C, D\}} \frac{x_{n-1}^{2}+x_{n} x_{n-1}+x_{n}}{x_{n-1}^{2}+x_{n} x_{n-1}+x_{n}} \\
& =\frac{\max \{\beta, \gamma, \delta\}}{\min \{B, C, D\}}=U .
\end{aligned}
$$

By using a similar method as in the proof of Theorem 10 one can prove the following result.

Theorem 11 Consider (1), where $B=\beta=0$, and all other coefficients are positive, subject to the condition

$$
\frac{|\gamma-C \bar{x}|(U+\bar{x})+|\delta-D \bar{x}|}{C L^{2}+D L}<1
$$

where $L=\frac{\min \{\gamma, \delta\}}{\max \{C, D\}}, U=\frac{\max \{\gamma, \delta\}}{\min \{C, D\}}$. Then $\bar{x}$ is globally asymptotically stable.

Proof Now, we have

$$
x_{n+1}=\frac{\gamma x_{n-1}^{2}+\delta x_{n}}{C x_{n-1}^{2}+D x_{n}} \geq \frac{\min \{\gamma, \delta\}}{\max \{C, D\}} \frac{x_{n-1}^{2}+x_{n}}{x_{n-1}^{2}+x_{n}}=\frac{\min \{\gamma, \delta\}}{\max \{C, D\}}=L>0,
$$

and

$$
x_{n+1}=\frac{\gamma x_{n-1}^{2}+\delta x_{n}}{C x_{n-1}^{2}+D x_{n}} \leq \frac{\max \{\gamma, \delta\}}{\min \{C, D\}} \frac{x_{n-1}^{2}+x_{n}}{x_{n-1}^{2}+x_{n}}=\frac{\max \{\gamma, \delta\}}{\min \{C, D\}}=U .
$$

Remark 1 Equation (1), where $D=\delta=0$ and all other coefficients are positive, reduces to the well-known equation (4) which was studied in great detail in [11,17] and for which we have shown that the unique equilibrium is globally asymptotically stable if and only if is locally asymptotically stable, that is, if and only if condition (i) of Theorem 3 holds. This result is certainly better than the global asymptotic result we derive from Corollaries 1 and 2 .

Remark 2 Equation (1), where either $B=0$ or $C=0$, and all other coefficients are positive, can be treated with Corollary 2 and the global asymptotic stability of the equilibrium (whenever it exists) follows from condition (6) in the interval $\left[\min \left\{\bar{x}, x_{-1}, x_{0}\right\}\right.$, $\left.\max \left\{\bar{x}, x_{-1}, x_{0}\right\}\right]$ when $\min \left\{\bar{x}, x_{-1}, x_{0}\right\}>0$, that is, when $x_{-1} x_{0}>0$. Similarly, (1), where exactly one of the coefficients $\beta, \gamma$ or $\delta$ is zero, and all other coefficients are positive, can be treated with Corollary 2 and the global asymptotic stability of the equilibrium follows 
from condition (6) in the interval $\left[\min \left\{\bar{x}, x_{-1}, x_{0}\right\}, \max \left\{\bar{x}, x_{-1}, x_{0}\right\}\right]$ when $\min \left\{\bar{x}, x_{-1}, x_{0}\right\}>0$, that is, when $x_{-1} x_{0}>0$. In this case $\max \left\{\bar{x}, x_{-1}, x_{0}\right\}$ can be replaced by $U=\frac{\max \{\beta, \gamma, \delta\}}{\min \{B, C, D\}}$.

\section{Competing interests}

The authors declare that they have no competing interests.

\section{Authors' contributions}

Each of the authors, MK, EP, and ES, contributed to each part of this work equally and read and approved the final version of the manuscript.

\section{Author details}

${ }^{1}$ Department of Mathematics, University of Rhode Island, Kingston, Rhode Island 02881-0816, USA. ²Department of Mathematics, University of Sarajevo, Sarajevo, Bosnia and Herzegovina. ${ }^{3}$ High School, Cazin, Bosnia and Herzegovina.

\section{Acknowledgements}

The authors are grateful to two anonymous referees for a number of suggestions, which have improved the quality of the exposition of the results.

Received: 24 December 2013 Accepted: 5 February 2014 Published: 18 Feb 2014

\section{References}

1. Amleh, AM, Camouzis, E, Ladas, G: On the dynamics of a rational difference equation, Part I. Int. J. Differ. Equ. 3, 1-35 (2008)

2. Amleh, AM, Camouzis, E, Ladas, G: On the dynamics of a rational difference equation, Part II. Int. J. Differ. Equ. 3, 195-225 (2008)

3. Kulenović, MRS, Merino, O: A global attractivity result for maps with invariant boxes. Discrete Contin. Dyn. Syst., Ser. B 6. 97-110 (2006)

4. Janowski, EA, Kulenović, MRS: Attractivity and global stability for linearizable difference equations. Comput. Math Appl. 57, 1592-1607 (2009)

5. Kent, CM, Sedaghat, $\mathrm{H}$ : Global attractivity in a quadratic-linear rational difference equation with delay. J. Differ. Equ. Appl. 15, 913-925 (2009)

6. Kent, CM, Sedaghat, $\mathrm{H}$ : Global attractivity in a rational delay difference equation with quadratic terms. J. Differ. Equ. Appl. 17, 457-466 (2011)

7. Sedaghat, $\mathrm{H}$ : Global behaviours of rational difference equations of orders two and three with quadratic terms. J. Differ. Equ. Appl. 15, 215-224 (2009)

8. Elaydi, SN: An Introduction to Difference Equations. 3rd edn. Undergraduate Texts in Mathematics. Springer, New York (2005)

9. Kulenović, MRS, Merino, O: Global bifurcations for competitive systems in the plane. Discrete Contin. Dyn. Syst., Ser. B 12, 133-149 (2009)

10. Brett, A, Kulenović, MRS: Basins of attraction of equilibrium points of monotone difference equations. Sarajevo J. Math. 5, 211-233 (2009)

11. Kulenović, MRS, Ladas, G: Dynamics of Second Order Rational Difference Equations with Open Problems and Conjectures. Chapman \& Hall/CRC, Boca Raton (2001)

12. Kulenović, MRS, Merino, O: Discrete Dynamical Systems and Difference Equations with Mathematica. Chapman \& Hall/CRC, Boca Raton (2002)

13. Kulenović, MRS, Merino, O: Invariant manifolds for competitive discrete systems in the plane. Int. J. Bifurc. Chaos Appl. Sci. Eng. 20, 2471-2486 (2010)

14. Sedaghat, H: Nonlinear Difference Equations: Theory with Applications to Social Science Models. Mathematical Modelling: Theory and Applications, vol. 15. Kluwer Academic, Dordrecht (2003)

15. Allen, L: An Introduction to Mathematical Biology, Pearson Education, Upper Saddle River (2006)

16. Camouzis, E, Ladas, G: Dynamics of Third-Order Rational Difference Equations with Open Problems and Conjectures. Advances in Discrete Mathematics and Applications, vol. 5. Chapman \& Hall/CRC, Boca Raton (2008)

17. Kulenović, MRS, Ladas, $G$, Sizer, W: On the recursive sequence $x_{n+1}=\left(\alpha x_{n}+\beta x_{n-1}\right) /\left(\gamma x_{n}+\delta x_{n-1}\right)$. Math. Sci. Res. Hot-Line 2, 1-16 (1998)

18. Enciso, EG, Sontag, ED: Global attractivity, I/O monotone small-gain theorems, and biological delay systems. Discrete Contin. Dyn. Syst. 14, 549-578 (2006)

19. DiPippo, M, Janowski, EJ, Kulenović, MRS: Global stability and attractivity of second order quadratic fractiona difference equation (to appear)

20. Kalabušić, S, Kulenović, MRS, Pilav, E: Global dynamics of a competitive system of rational difference equations in the plane. Adv. Differ. Equ. 2009, Article ID 132802 (2009)

21. Kalabušić, S, Kulenović, MRS, Pilav, E: Dynamics of a two-dimensional system of rational difference equations of Leslie-Gower type. Adv. Differ. Equ. 2011, Article ID 29 (2011)

22. Elaydi, SN: Discrete Chaos: With Applications in Science and Engineering, 2nd edn. Chapman \& Hall/CRC, Boca Raton (2008)

23. Agarwal, RP, Pituk, M: Asymptotic expansions for higher-order scalar difference equations. Adv. Differ. Equ. 2007, Article ID 067492 (2007)

24. Kalabušić, S, Kulenović, MRS: Rate of convergence of solutions of rational difference equation of second order. Adv. Differ. Equ. 2004(2), 121-139 (2004)

25. Pituk, M: More on Poincare's and Perron's theorems for difference equations. J. Differ. Equ. Appl. 8(3), 201-216 (2002) 
10.1186/1687-1847-2014-68

Cite this article as: Kulenović et al.: Local dynamics and global attractivity of a certain second-order quadratic fractional difference equation. Advances in Difference Equations 2014, 2014:68

Submit your manuscript to a SpringerOpen ${ }^{\circ}$ journal and benefit from:

- Convenient online submission

- Rigorous peer review

- Immediate publication on acceptance

- Open access: articles freely available online

- High visibility within the field

- Retaining the copyright to your article

Submit your next manuscript at $\gg$ springeropen.com 